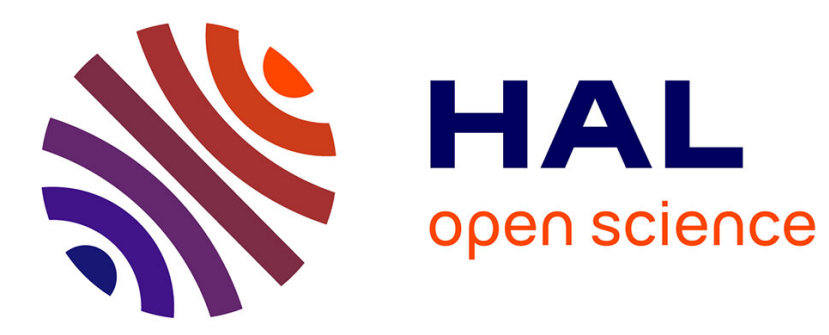

\title{
Investigation of the interaction of a turbulent impinging jet and a heated, rotating disk
}

\author{
Remi Manceau, R. Perrin, M. Hadziabdic, S. Benhamadouche
}

\section{To cite this version:}

Remi Manceau, R. Perrin, M. Hadziabdic, S. Benhamadouche. Investigation of the interaction of a turbulent impinging jet and a heated, rotating disk. Physics of Fluids, 2014, 26 (3), pp.30. 10.1063/1.4867380 . hal-01051796

\section{HAL Id: hal-01051796 https://hal.science/hal-01051796}

Submitted on 18 Dec 2015

HAL is a multi-disciplinary open access archive for the deposit and dissemination of scientific research documents, whether they are published or not. The documents may come from teaching and research institutions in France or abroad, or from public or private research centers.
L'archive ouverte pluridisciplinaire HAL, est destinée au dépôt et à la diffusion de documents scientifiques de niveau recherche, publiés ou non, émanant des établissements d'enseignement et de recherche français ou étrangers, des laboratoires publics ou privés. 


\title{
Investigation of the interaction of a turbulent impinging jet and a heated, rotating disk
}

\author{
R. Manceau, ${ }^{1,2, a)}$ R. Perrin, ${ }^{2}$ M. Hadžiabdić, ${ }^{3}$ and S. Benhamadouche ${ }^{4}$ \\ ${ }^{1}$ Department of Applied Mathematics, CNRS-University of Pau-Inria, avenue de l'université, \\ 64013 Pau, France \\ ${ }^{2}$ Department of Fluids, Heat Transfer, Combustion, Institute Pprime, CNRS-University of \\ Poitiers-ENSMA, Boulevard Marie et Pierre Curie, 86962 Futuroscope-Chasseneuil, France \\ ${ }^{3}$ Faculty of Engineering and Natural Sciences, International University of Sarajevo, \\ Hrasnička cesta 15, 71210 Sarajevo, Bosnia and Herzegovina \\ ${ }^{4}$ Department MFEE, EDF R\&D, 6, quai Watier, 78401 Chatou, France
}

(Received 28 July 2013; accepted 10 February 2014; published online 12 March 2014)

The case of a turbulent round jet impinging perpendicularly onto a rotating, heated disc is investigated, in order to understand the mechanisms at the origin of the influence of rotation on the radial wall jet and associated heat transfer. The present study is based on the complementary use of an analysis of the orders of magnitude of the terms of the mean momentum and Reynolds stress transport equations, available experiments, and dedicated Reynolds-averaged Navier-Stokes computations with refined turbulence models. The Reynolds number $R e_{j}=14500$, the orifice-to-plate distance $H=5 D$, where $D$ is the jet-orifice diameter, and the four rotation rates were chosen to match the experiments of Minagawa and Obi ["Development of turbulent impinging jet on a rotating disk," Int. J. Heat Fluid Flow 25, 759-766 (2004)] and comparisons are made with the Nusselt number distribution measured by Popiel and Boguslawski ["Local heat transfer from a rotating disk in an impinging round jet," J. Heat Transfer 108, 357-364 (1986)], at a higher Reynolds number. The overestimation of turbulent mixing in the free-jet before the impact on the disk is detrimental to the prediction of the impingement region, in particular of the Nusselt number close to the symmetry axis, but the self-similar wall jet developing along the disk is correctly reproduced by the models. The analysis, experiments, and computations show that the rotational effect do not directly affect the outer layer, but only the inner layer of the wall jet. A noteworthy consequence is that entrainment at the outer edge of the wall jet is insensitive to rotation, which explains the dependence of the wall-jet thickness on the inverse of the non-dimensional rotation rate, observed in the experiments and the Reynolds stress model computations, but not reproduced by the eddy-viscosity models, due to the algebraic dependence to the mean flow. The analysis makes moreover possible the identification of a scenario for the appearance of rotational effects when the rotation rate is gradually increased. For weak rotation rates, the rotation-induced boundary layer appears but does not break the selfsimilar solution observed for the case without rotation. For intermediate rotation rates, the production of the azimuthal Reynolds stress becomes much stronger than other components, leading to a complete modification of the turbulence anisotropy which is reproduced only by Reynolds stress models. For strong rotation rates, centrifugal effects dominate, leading to an acceleration and thinning of the jet, and consequently an increase of turbulent production and heat transfer, reproduced by all the turbulence models. (C) 2014 AIP Publishing LLC. [http://dx.doi.org/10.1063/1.4867380]

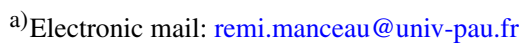




\section{INTRODUCTION}

Impinging jet problems, ranging from axisymmetric, plane, to more complex configurations, have been extensively investigated, experimentally and numerically, because of their significance for a range of industrial applications, for turbine blade cooling, drying or heating processes, cooling of electronic devices, etc. It is widely recognized that turbulent impinging jets, despite their geometric simplicity, contain a complex physics, which makes them attractive for studying various features of turbulence dynamics, its interaction with the impinged wall and resulting effects on heat transfer.

The physical composition of impinging jets is not unique since it depends upon a number of parameters, such as Reynolds number, orifice-to-plate distance, inflow turbulence, and orifice shape and profile. A common perception of a simple round impinging jet implies three distinct regions through which fluid passes, as depicted in Fig. 1 for the case of a rotating plate-the free jet, the impingement region, and the radial wall-jet region, each featured by different prevailing turbulence dynamics and each governed by some specific generic turbulence mechanisms. One of the main processes that influence the impinging jet dynamics and heat transfer is the growing unstable edge shear layer that surrounds the free jet core.

For the turbulence modeling community, simple configurations, such as the axisymmetric jet impinging on a flat plate, have served as fundamental test cases for the investigation of the performance of turbulence models, since they are one of the wall-bounded flows not only driven by shear, but also by plane strain, streamline curvature, and complex wall-blocking effects. In the impingement region, the occurrence, close to the wall, of negative production of turbulent kinetic energy, balanced by pressure diffusion, ${ }^{1}$ is impossible to predict by Reynolds-Averaged Navier-Stokes (RANS) models based on the simple eddy-viscosity concept. Because of such complex effects, impinging jets are well suited for testing the performance of RANS turbulence models.

The case of a jet impinging on a rotating disk has received less attention, despite its relevance to cooling processes in many industrial applications, such as the cooling of bearings, gears, harddisk drives, gas turbines disks, and alternators of wind generators. ${ }^{2-4}$ Some authors performed experimental measurement, mainly focusing on the impingement of a non-centered jet, ${ }^{2,5,6}$ a case pertaining to the cooling of gas turbine disks. Even in the case of a centered jet, the flow still implies a complex interaction between the radial wall jet surrounding the impingement region and

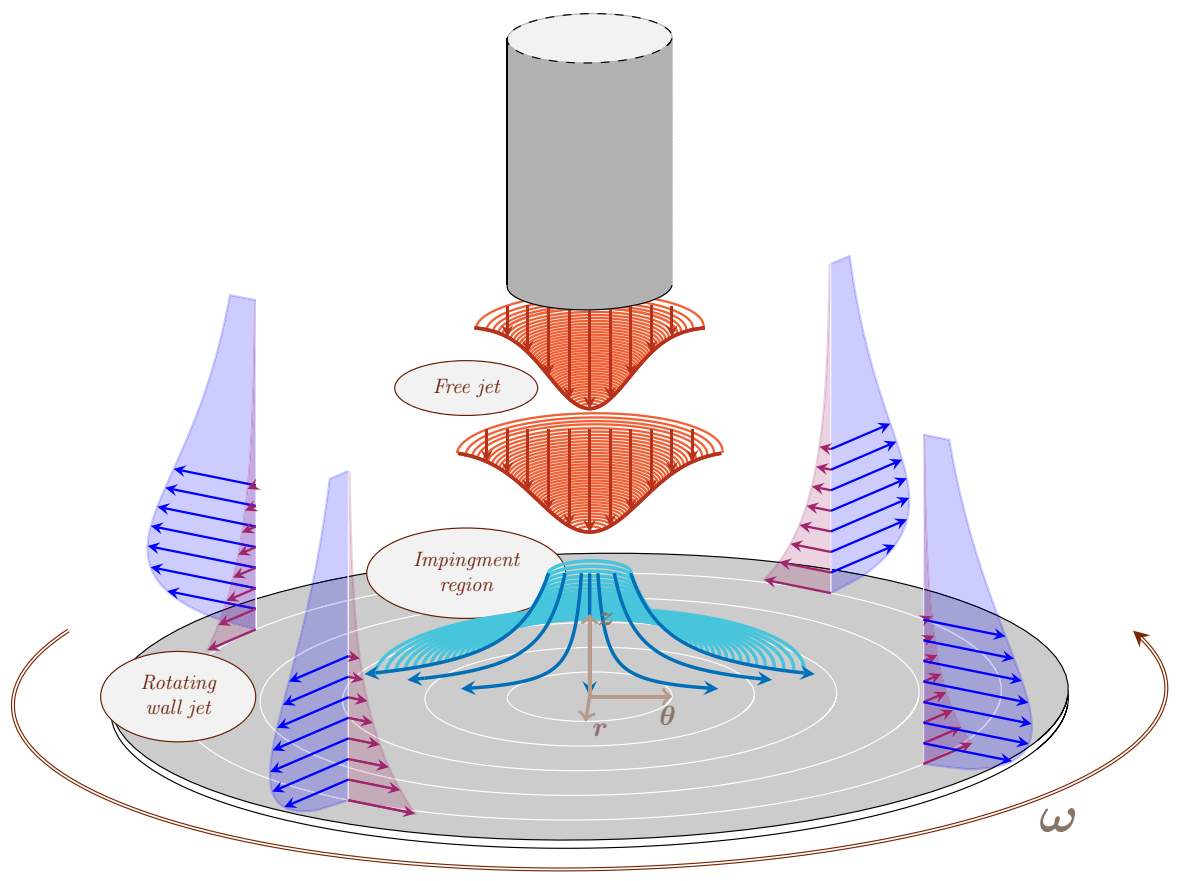

FIG. 1. Artist view of the different regions of the flow. 
the azimuthal boundary layer, or Ekman layer, due to the rotation of the disk, as shown in Fig. 1. Extending the method of von Kármán ${ }^{7}$ for the flow over a rotating disk in still air, Truckenbrodt ${ }^{8}$ was able to evaluate the modification of the orientation of the flow due to a uniform current impinging on the disk (for an extensive review of these early studies, see Dorfman ${ }^{9}$ ). Flow field measurements of the case of a localized jet impinging at the center of the disk were performed by Itoh and $\mathrm{Okada}^{10}$ and Minagawa and Obi, ${ }^{11}$ and heat transfer measurements by Chen, Lee, and $\mathrm{Wu},{ }^{2}$ Popiel and Boguslawski, ${ }^{6}$ and Astarita and Cardone ${ }^{12}$ (see, for instance, the recent review by Harmand, Pellé, Poncet, and Shevchuk ${ }^{4}$ ). The present paper aims at extending the very limited knowledge and understanding of the case of a centered jet, in particular as concerns the mechanisms of the migration from a self-similar axisymmetric solution due to the spreading of the impinging jet to a rotation-dominated flow. This collaborative work, initiated in the frame of the workshop organized at the University of Technology of Graz, Austria, under the auspices of the Special Interest Group 15 turbulence modelling of the European Research Community On Flow, Turbulence and Combustion (ERCOFTAC), ${ }^{13}$ has led to a deeper understanding of this complex flow, thus providing an excellent example of cross-fertilization of analytical, experimental, and numerical studies.

\section{PRESENTATION OF THE CONFIGURATION AND THE METHODS}

The present investigation is primarily based on the experiments dedicated to the measurement of the flow field performed by Minagawa and Obi. ${ }^{11}$ Comparisons with heat transfer measurements, ${ }^{6}$ at a higher Reynolds number, are also presented. The configuration and the lines along which the results will be extracted for comparison with the experimental profiles are shown in Fig. 2. The flow is issuing from a circular pipe of diameter $D$, at a distance $H=5 D$ from the disk with the bulk velocity $U_{j}$. The radius of the disk is $R=6 D$ and the Reynolds number is $R e_{j}=U_{j} D / v$ $=14500$. Several rotation rates were investigated by Minagawa and Obi ${ }^{11}$ using Laser Doppler Anemometry (LDA): $\omega D / U_{j}=0,0.12,0.24$, and 0.48 . The rotation of the disk onto which the jet is impinging is at the origin of a rotation-induced boundary layer that leads to a skewing of the wall-jet created around the impingement point (see Fig. 1). Therefore, this case provides a example of two-dimensional, axisymmetric flow $(\partial / \partial \theta=0)$ subject to a complex, six-component strain, pertinent to the investigation of effects present in complex three-dimensional flows.

The computations were performed using two in-house, unstructured, finite-volume computational codes, T-FlowS ${ }^{14,15}$ developed at Delft University of Technology and the open-source (www.code-saturne.org) Code_Saturne developed at EDF. ${ }^{16}$ Both codes utilize the cell-centered collocated arrangement. A second-order upwind-biased differencing scheme is used for the
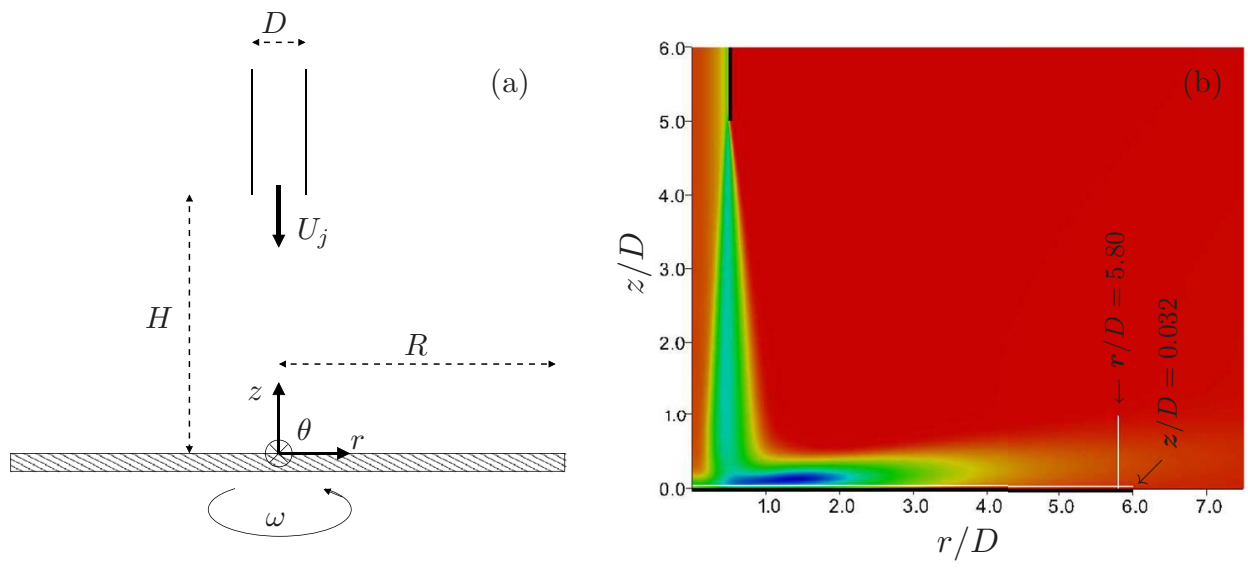

FIG. 2. (a) Schematic view of the geometry. (b) Lines along which the results will be extracted, in order to compare with the experimental data of Minagawa and $\mathrm{Obi}^{11}$ (white lines). To better illustrate the location of these profiles in the flow, the turbulent energy field given by the EB-RSM is shown (case without rotation). Walls (nozzle and disk) are represented by thick black lines. 
convective terms in the governing equations in T-FlowS and the pressure is solved with a SIMPLE algorithm. In Code_Saturne, a fully centered scheme is used for the velocity components and an upwind scheme for the turbulent quantities. The temperature, considered as a passive scalar, is solved with a centered scheme and the pressure is corrected with a SIMPLEC algorithm.

As usual in the ERCOFTAC SIG-15 workshop series, the groups computed the cases independently using different models, and performed grid refinement studies to check the numerical accuracy. The $\zeta-f$ model $^{17}$ was used in T-FlowS (International University of Sarajevo); the $k-\omega-$ $\mathrm{SST}^{18}$ and $\varphi-f^{19}$ models were used in Code_Saturne (EDF); the Elliptic-Blending Reynolds stress model $^{20,21}$ (EB-RSM), which is an extension to the near-wall region of the SSG model, ${ }^{22}$ was also used in Code_Saturne (Institute Pprime). Eddy-viscosity models were associated with the simple gradient diffusion hypothesis model for the turbulent heat fluxes, while the Reynolds stress model was associated with the generalized gradient diffusion hypothesis model.

\section{QUALITATIVE COMPARISON OF MODEL PERFORMANCE}

As will be detailed in Secs. IV and V, the flow in the wall jet region (say $r / D>2.5$ ) can be divided into two layers: the outer layer and the inner layer that essentially behave as a free jet and a boundary layer, respectively. The boundary layer induced by the rotation of the disk is too thin to directly interact with the outer layer (see Sec. V), such that, in order to have a global picture of the influence of rotation on the flow, Minagawa and $\mathrm{Obi}^{11}$ extracted profiles in the inner layer, at $z / D=0.032$ (see Fig. 2(b)). The mean radial velocity $V_{r}$ and the rms radial velocity $v_{r}^{\prime}$ for four different rotation rates are shown in Fig. 3 for the experiments and the four turbulence models. The detailed measurement of Minagawa and $\mathrm{Obi}^{11}$ for the mean field and Reynolds stresses are at the four rotation rates $\omega D / U_{j}=0,0.12,0.24$, and 0.48 , and the computations were performed for these rates. The profiles in the inner layer at $z / D=0.032$ corresponds to slightly different rates $\omega D / U_{j}=$ $0,0.15,0.30$, and 0.45 , but the comparison remains relevant from a qualitative point of view.

The experiments show that the flow can be divided into three characteristic regions: the impingement region, dominated by plane strain, between the symmetry axis and the location of the mean velocity peak; the region of strong deflection of the streamlines, approximately located between the mean velocity peak and $r / D=2.5$; the wall jet, after $r / D=2.5$, in which the radial velocity, because of the axisymmetry of the flow, decreases in $1 / r$ in the absence of rotation, a behavior analyzed in detail in Sec. IV.

It can be seen in Fig. 3 that all the models fail to correctly predict the peak in the radial distribution of the mean radial velocity, the best prediction being given by the $\varphi-f$ model, and the worst by the Reynolds-stress model. This discrepancy is linked to the overestimation of the spreading of the free jet before the impingement on the disk, which is investigated in detail in Sec. IV.

The EB-RSM provides a rather satisfactory profile of the rms fluctuation $v_{r}^{\prime}$, especially for $r / D$ $>2.5$. However, in the region between $r / D=1$ and $r / D=2.5$, the experiment show a plateau of $v_{r}^{\prime}$ that is not reproduced by the models. This plateau is located where the free-jet shear layer impinges on the disk, and could be the footprint of the large-scale unsteadiness induced by the impact of coherent structures, which cannot be reproduced by RANS models.

The misprediction of the distributions of the radial rms velocity by the eddy-viscosity models is not surprising since they are based on the Boussinesq relation. The over-prediction of $v_{r}^{\prime}$ by the $\varphi-f$ model in the impingement region can be related to the absence of production limiter, contrary to the $\zeta-f$ and $k-\omega-$ SST models that both underestimate $v_{r}^{\prime}$. The latter two models give comparable results for $v_{r}^{\prime}$ with a slight superiority of the $\zeta-f$ model in the impingement region, where the $k-\omega-\mathrm{SST}$ model exhibits a pronounced plateau at a low level, close to the centerline, and a sharp increase starting at $r / D=0.5$. These discrepancies between the models have strong implications for the comparison of their heat transfer predictions in the impingement region, as can be seen in Fig. 4, which shows the Nusselt number $\mathrm{Nu}$ distributions obtained in the case without rotation, with

$$
N u=\frac{\dot{q}_{w} D}{\lambda\left(T_{w}-T_{\infty}\right)},
$$



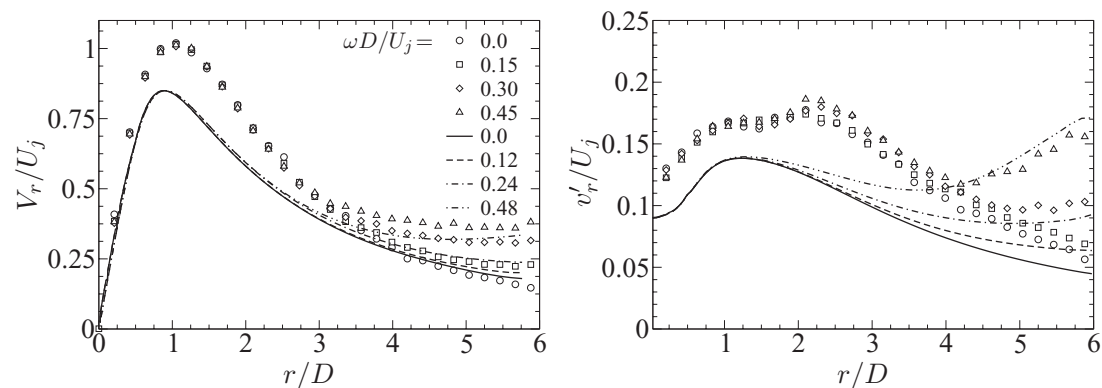

(a)
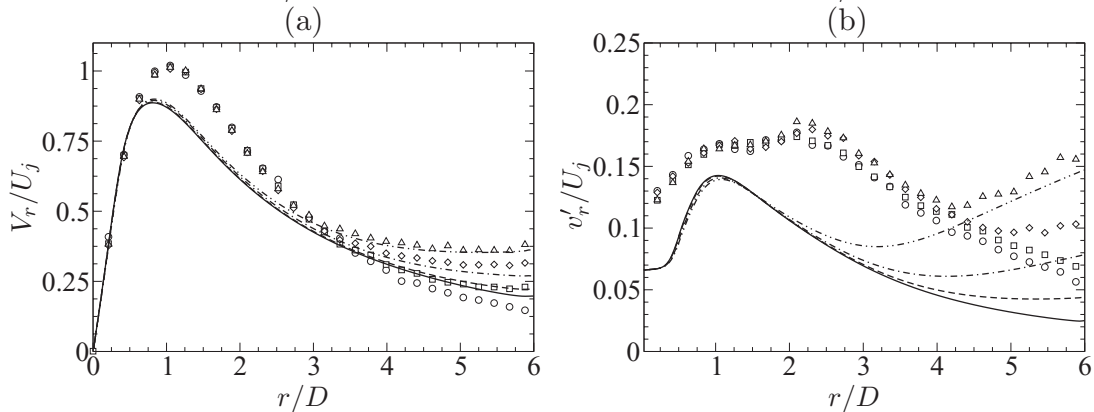

(c)

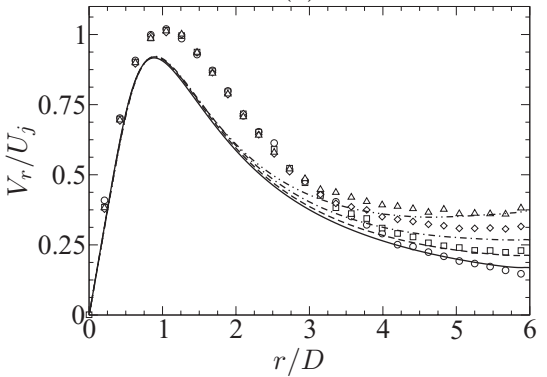

$(\mathrm{d})$

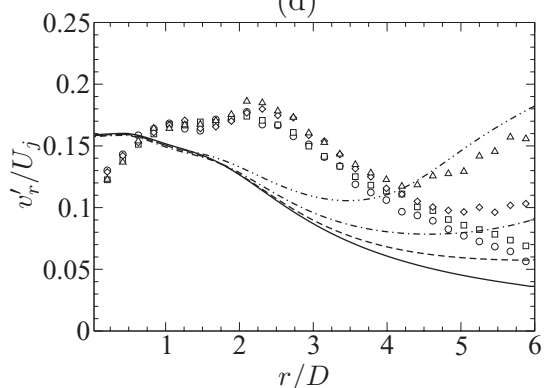

(e)

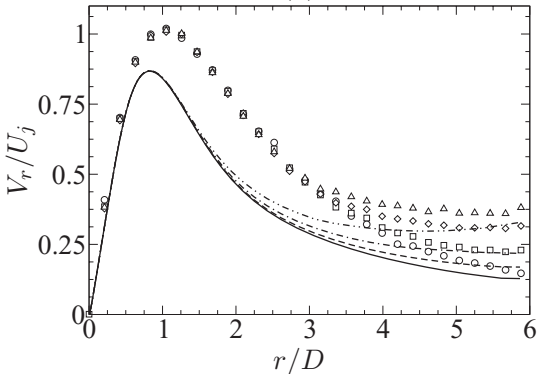

(g)

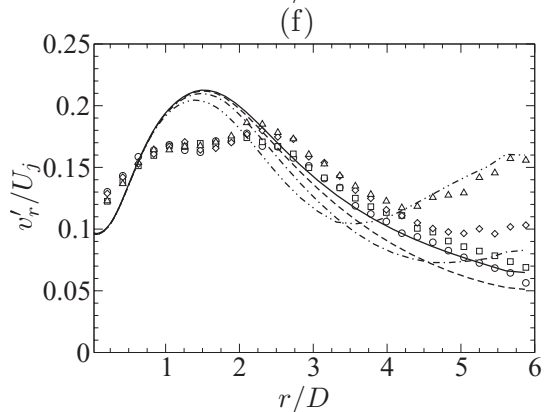

(h)

FIG. 3. Profiles of the mean radial velocity (left) and the rms radial velocity (right) at $z / D=0.032$. (a) and (b) $\zeta-f$; (c) and (d) $k-\omega-\mathrm{SST}$; (e) and (f) $\varphi-f$; (g) and (h) EB-RSM.

where $T_{w}$ and $T_{\infty}$ are the temperature at the wall and the ambient temperature, respectively, and $\dot{q}_{w}$ is the imposed wall heat flux. The well-known sensitivity of the heat transfer to the level of turbulence intensity observed in the impingement region is confirmed. The striking discrepancy between the two $\overline{v^{2}}-f$-type models $(\varphi-f$ and $\zeta-f)$ can only be explained by the presence of the production limiter in the $\zeta-f$ version, induced by the realizability constraint imposed on the length and time scale. ${ }^{24}$ As will be shown in Sec. IV A, the expansion rate of the free jet issuing from the nozzle is overestimated by all the models, such that impingement occurs after the end of the potential core, contrary to what is observed in the experiments. In the $k-\omega-\mathrm{SST}$ and $\zeta-f$ models, production limiters are used, such that the intensity of the turbulent fluctuations is significantly reduced in this region, enabling the 


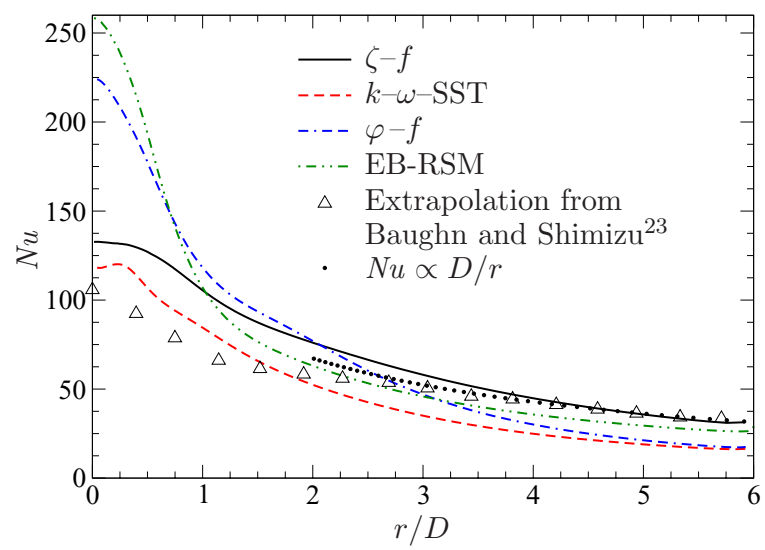

FIG. 4. Nusselt number distribution on the disk. The dotted line represents a least-square fit in the region $r / D>3$ of the extrapolated experimental data.

models to provide lower Nusselt number levels, but drawing a conclusion as concerns the accuracy of these predictions is difficult, since heat transfer measurements at the present Reynolds number $R e_{j}=14500$ and nozzle-to-plate distance are not available. However, in order to provide at least an estimate of the expected level of Nusselt number, the data of Baughn and Shimizu, ${ }^{23}$ at $R e_{j}=$ 23000 , have been extrapolated assuming that the Nusselt number scales with $R e_{j}^{3 / 4}$, as will be found in Sec. IV B 3. $k-\omega-$ SST and $\zeta-f$ models give comparable results and are close to the extrapolated data.

As can be seen in Fig. 3, when the disk is rotated, the experimental data show that the mean velocity and stress fields are not significantly affected up to $r / D=2.5$ : for the rotation rates under consideration, the flow in the region $r / D<2.5$ remains dominated by pressure and convection terms. This is the reason why the remaining of the present article mainly focuses on the wall jet region $(r / D$ $>2.5$ ). In this region, the influence of centrifugal acceleration increases with the rotation rate and the distance to the symmetry axis, such that the mean and fluctuating velocities gradually deviate from their profiles in the non-rotational case.

Figure 5 shows profiles, taken along the $z$-axis (normal to the wall), of the radial mean velocity profiles close to the extremity of the disk $(r / D=5.8)$, for the four rotation rates. It can be seen that the acceleration of the flow induces a thinning of the wall jet, by the virtue of mass conservation. Note that for the non-rotational case, the under-prediction of the peak velocity by the second moment closure is to be linked to the global underestimation of the mean velocity shown in Fig. 3. The discrepancies between the predictions of the models are attenuated when the rotation rate is increased, since, as

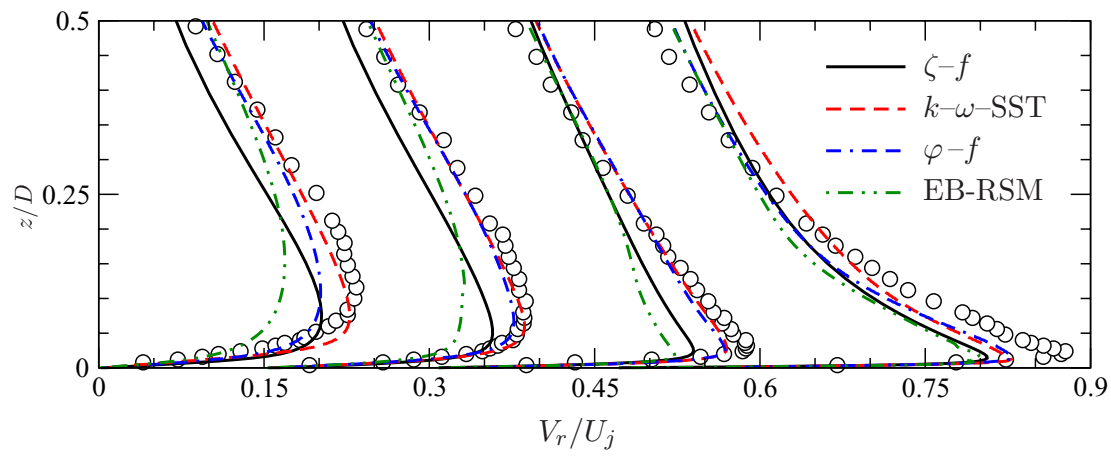

FIG. 5. Evolution of the radial mean velocity profiles at $r / D=5.80$ with increasing rotation rates (from the left to the right: $\omega D / U_{j}=0,0.12,0.24$, and 0.48 ). For the sake of clarity, profiles are shifted by steps of 0.15 along the horizontal axis. 


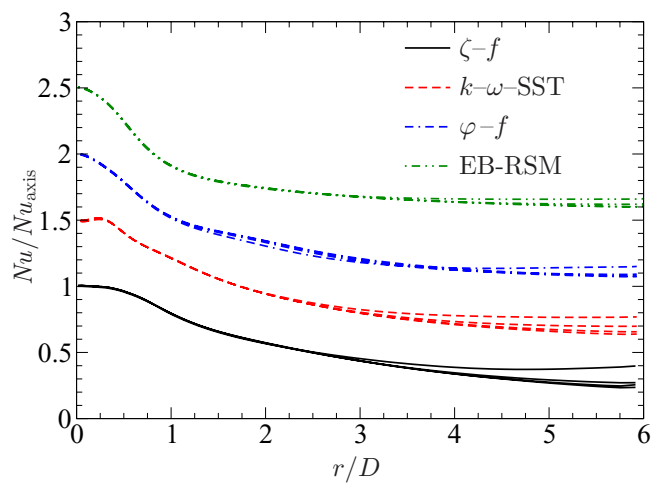

FIG. 6. Evolution with the rotation rate of the Nusselt number distribution on the disk (profiles are made non-dimensional by the value at the stagnation point and shifted for clarity).

will be shown in Sec. V, the centrifugal acceleration becomes dominant in the momentum equation, such that the mean flow prediction is independent of turbulence modeling.

The modification of the mean velocity gradient $\partial V_{r} / \partial z$ due to both the acceleration and the thinning of the wall jet implies an increase of turbulent production, which generates strong radial fluctuations $v_{r}^{\prime}$, as can be seen in Fig. 3. Such an effect is captured by all the models, even eddyviscosity models that include the direct influence of the shear. However, it can be seen that eddyviscosity models give a much too early $(r / D \approx 2$ rather than $r / D \approx 4)$ increase of $v_{r}^{\prime}$, which can be traced to the fact that the modeled production is explicitly quadratic in the shear rather than linear. Indeed, as will be demonstrated in Sec. V C 2, the dominant term in the turbulence production is

$$
2 \overline{v_{r} v_{z}} \frac{\partial V_{r}}{\partial z}
$$

such that the intensification of the shear is directly felt through the presence of $\partial V_{r} / \partial z$ in Eq. (2), and indirectly through the increase of $\overline{v_{r} v_{z}}$, whose dominant production term

$$
\overline{v_{z}^{2}} \frac{\partial V_{r}}{\partial z}
$$

also involves $\partial V_{r} / \partial z$. Therefore, turbulence production is indeed quadratic in the shear $\partial V_{r} / \partial z$, but only after a sufficient distance necessary for $\overline{v_{r} v_{z}}$ to respond to the modification of this shear. On the contrary, with eddy-viscosity models, in which the Reynolds stress is modeled by

$$
\overline{v_{i} v_{j}}=-2 v_{t} S_{i j}+\frac{2}{3} k \delta_{i j},
$$

production reads

$$
P=2 v_{t} S_{i j} S_{i j}
$$

such that the response is immediately quadratic, through the dominant production term

$$
v_{t}\left(\frac{\partial V_{r}}{\partial z}\right)^{2}
$$

Finally, Fig. 6 shows the evolution of the Nusselt number distribution with the rotation rate. The models are able to predict the heat transfer enhancement due to the increase of turbulent mixing when the disk is rotated. To the authors knowledge, no experimental data are available at this Reynolds number. However, the scaling properties of heat transfer with rotation rate are investigated in Sec. V, in comparison with data from Popiel and Boguslawski ${ }^{6}$ at higher Reynolds numbers. 

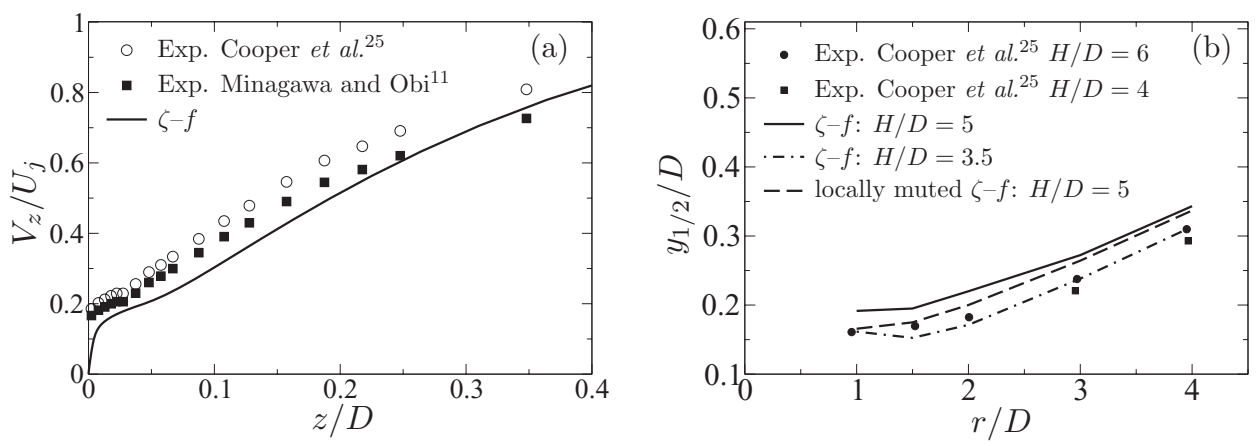

FIG. 7. (a) Centerline velocity. (b) Half-thickness of the wall jet (defined by the distance where $50 \%$ of maximum radial velocity $V_{r \max }$ is reached).

\section{ANALYSIS OF THE NON-ROTATIONAL CASE}

\section{A. Free jet}

As shown in Fig. 3, all the models fail to correctly predict the peak in the radial distribution of the mean radial velocity in the wall-jet at $z / D=0.032$. This result is linked to the over-prediction of the mixing in the free-jet shear-layer, which leads to an overestimation of the jet growth and consequently to a reduction of the maximum velocities in the wall-jet region due to the continuity constraint, as shown in Fig. 7. As a consequence, the wall-jet half-width is larger than experimentally measured, as is revealed by Fig. 7(b).

In order to further investigate the above scenario, two numerical experiments have been conducted. First, a computation was performed for the reduced orifice-to-plate ratio $H / D=3.5$. Second, for the same geometry $(H / D=5)$, in order to drastically, albeit artificially, reduce the mixing in the free jet, the turbulence model $(\zeta-f)$ was switched off in the momentum equation in the region $1.0<z / D<5.0$ (the method is denoted by locally muted $\zeta-f$ in Figs. 7 and 8). Figure 7(b) shows that the thickness of the wall-jet is significantly reduced in both cases. The fact that, after some distance in the radial direction, the growth rate is the same for the 3 computations, as well as the fact that, in Fig. 8(a), the two numerical experiments give a significantly improved prediction of the peak of the mean radial velocity confirm that the over-predicted mixing in the free-jet shear layer is the main cause for the poor prediction of the mean velocity field in the wall-jet region. This problem can possibly be related to the well-known plane jet/round jet anomaly. ${ }^{26}$
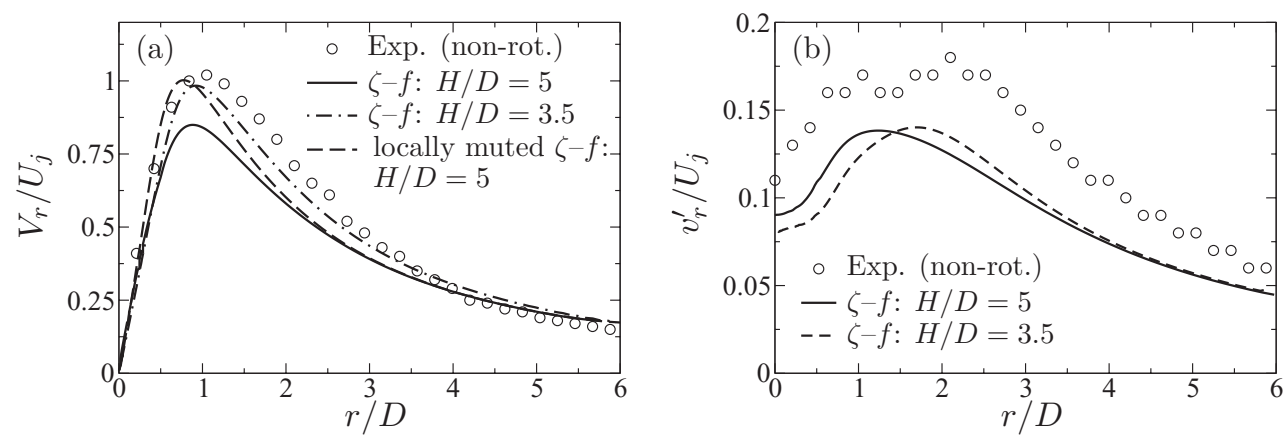

FIG. 8. Comparison of the profiles at $z / D=0.032$ obtained from the computations and the experiments by Minagawa and Obi. ${ }^{11}$ (a) Mean radial velocity. (b) rms radial velocity. 


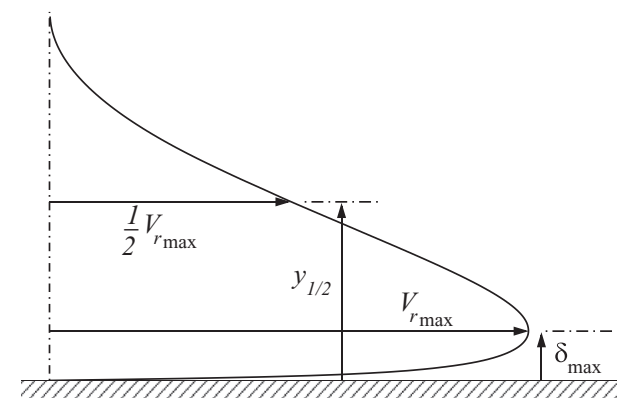

FIG. 9. Definition of the standard velocity and length scales used to describe the wall jet.

\section{B. Structure of the wall jet}

In the absence of rotation, the wall jet developing along the disk outside the impingement region (see Fig. 1) can be characterized by the maximum velocity $V_{r \max }$ and the jet thickness $\delta$. From the early work of Glauert, ${ }^{27}$ the standard definition of this thickness was the distance to the wall where the mean radial velocity in the outer layer reaches $\frac{1}{2} V_{r \max }$, generally denoted as $y_{1 / 2}$ (see Fig. 9), which was experimentally easy to measure (see the review paper of Launder and $\operatorname{Rodi}^{28}$ ). Other authors, in particular Minagawa and $\mathrm{Obi}^{11}{ }^{11}$ use $\delta_{\max }$, the distance to the wall where the maximal velocity is reached. In cases with rotation, where self-similarity is broken, the particular choice of the length scale definition becomes significant, and it will be shown that an integral definition, linked to the flow rate, is preferable, such that we define $\delta$ as

$$
\delta=\frac{1}{V_{r \max }} \int_{0}^{\infty} V_{r}(z) \mathrm{d} z .
$$

Due to entrainment, the flow rate $Q(r)=2 \pi r \delta V_{r \max }$ at some radial location $r$ is larger than the flow rate issuing from the pipe $Q_{j}=\pi \frac{D^{2}}{4} U_{j}$, and the ratio is denoted by $e=Q(r) / Q_{j}$, and called entrainment ratio hereafter. In order to simplify the analysis, a reduced thickness

$$
\delta^{\prime}=\frac{8 \delta}{e}
$$

is introduced. Using this definition, the relation $Q(r)=e Q_{j}$ yields

$$
V_{r \max }=\frac{U_{j} D^{2}}{r \delta^{\prime}} .
$$

Following the description of Launder and Rodi, ${ }^{28}$ based on the analysis initially proposed by Glauert, ${ }^{27}$ wall jets, be it plane, axisymmetric, or three-dimensional, can be seen as two-layer flows. The region located between the wall and $\delta_{\max }$, the location of the velocity maximum, called the inner region, exhibits characteristics close to those of a boundary layer; the region beyond $\delta_{\max }$, called the outer region, is similar to a free shear flow, and is governed by inviscid equations. A complete similarity of the flow can be achieved only if the inner and outer layers are in equilibrium, a hypothesis introduced by Glauert ${ }^{27}$ and partially supported by further experimental studies, ${ }^{29-31}$ which implies that the velocity scale of the inner layer, the friction velocity $u_{\tau}$, is proportional to the velocity scale of the outer layer, $V_{r \max } \cdot 31,32$

More recently, ${ }^{32,33}$ it was shown that this description is too simple to account for all the phenomena observed in this type of flows. Barenblatt $e t$ al. ${ }^{33}$ proposed the introduction of a third, intermediate layer, located between the inner and outer layers, in the region of the velocity maximum, and, in accordance with George et al. ${ }^{32}$ showed that the flow exhibits incomplete similarity only. However, such a refined analysis is beyond the scope of the present article, since it is not necessary for our purpose, i.e., the evaluation of the orders of magnitude of the different terms in the equations and the investigation of the influence of the superimposition of a rotation-induced boundary layer. 


\section{Governing equations}

In the case of the incompressible, stationary, axisymmetric flow under consideration, in the cylindrical coordinate frame defined in Fig. 2, the governing equations for the mean quantities are mass (10), momentum (11)-(13), and energy (14) conservation. The Reynolds average is used to decompose pressure, velocities, and temperature into mean $\left(P, V_{r}, V_{\theta}, V_{z}, T\right)$ and fluctuating $\left(p, v_{r}\right.$, $\left.v_{\theta}, v_{z}, \vartheta\right)$ parts

$$
\begin{gathered}
\frac{1}{r} \frac{\partial r V_{r}}{\partial r}+\frac{\partial V_{z}}{\partial z}=0 \\
V_{r} \frac{\partial V_{r}}{\partial r}+V_{z} \frac{\partial V_{r}}{\partial z}-\frac{V_{\theta}^{2}}{r}=-\frac{1}{\rho} \frac{\partial P}{\partial r}-\frac{\partial \overline{v_{r}^{2}}}{\partial r}-\frac{\overline{v_{r}^{2}}}{r}-\frac{\partial \overline{v_{r} v_{z}}}{\partial z}+\frac{\overline{v_{\theta}^{2}}}{r} \\
+v\left[\frac{1}{r} \frac{\partial}{\partial r}\left(r \frac{\partial V_{r}}{\partial r}\right)+\frac{\partial^{2} V_{r}}{\partial z^{2}}-\frac{V_{r}}{r^{2}}\right] \\
+v\left[\frac{1}{r} \frac{\partial}{\partial r}\left(r \frac{\partial V_{\theta}}{\partial r}\right)+\frac{\partial^{2} V_{\theta}}{\partial z^{2}}-\frac{V_{\theta}}{r^{2}}\right] \\
V_{r} \frac{\partial V_{\theta}}{\partial r}+V_{z} \frac{\partial V_{\theta}}{\partial z}+\frac{V_{r} V_{\theta}}{r}=-\frac{1}{\rho r} \frac{\partial P}{\partial \theta}-\frac{\partial \overline{v_{r} v_{\theta}}}{\partial r}-2 \frac{\overline{v_{r} v_{\theta}}}{r}-\frac{\partial \overline{v_{\theta} v_{z}}}{\partial z} \\
V_{r} \frac{\partial V_{z}}{\partial r}+V_{z} \frac{\partial V_{z}}{\partial z}=-\frac{1}{\rho} \frac{\partial P}{\partial z}-\frac{\partial \overline{v_{r} v_{z}}}{\partial r}-\frac{\overline{v_{r} v_{z}}}{r}-\frac{\partial \overline{v_{z}^{2}}}{\partial z} \\
+v\left[\frac{1}{r} \frac{\partial}{\partial r}\left(r \frac{\partial V_{z}}{\partial r}\right)+\frac{\partial^{2} V_{z}}{\partial z^{2}}\right] \\
V_{r} \frac{\partial T}{\partial r}+V_{z} \frac{\partial T}{\partial z}=-\frac{1}{r} \frac{\partial}{\partial r}\left(r \frac{\partial v_{r} \vartheta}{}\right)-\frac{\partial \overline{v_{z} \vartheta}}{\partial z}+\alpha\left[\frac{1}{r} \frac{\partial}{\partial r}\left(r \frac{\partial T}{\partial r}\right)+\frac{\partial^{2} T}{\partial z^{2}}\right] .
\end{gathered}
$$

The second moment of the velocity, the Reynolds stress tensor $\overline{v_{i} v_{j}}$, involved in these equations, satisfies the transport equation

$$
\begin{aligned}
\underbrace{V_{k} \frac{\partial \overline{v_{i} v_{j}}}{\partial x_{k}}}_{\mathcal{C}_{i j}}= & \underbrace{-\overline{v_{i} v_{k}} \frac{\partial V_{j}}{\partial x_{k}}-\overline{v_{j} v_{k}} \frac{\partial V_{i}}{\partial x_{k}}}_{P_{i j}}-\underbrace{2 v \overline{\frac{\partial v_{i}}{\partial x_{k}} \frac{\partial v_{j}}{\partial x_{k}}}}_{\varepsilon_{i j}} \\
& \underbrace{-\frac{1}{\rho} \overline{v_{i} \frac{\partial p}{\partial x_{j}}}-\frac{1}{\rho} \overline{v_{j} \frac{\partial p}{\partial x_{i}}}}_{\Pi_{i j}}+\underbrace{v \frac{\partial^{2} \overline{v_{i} v_{j}}}{\partial x_{k} \partial x_{k}}-\frac{\partial \overline{v_{i} v_{j} v_{k}}}{\partial x_{k}}}_{D_{i j}},
\end{aligned}
$$

where the tensors $\mathcal{C}_{i j}, P_{i j}, \varepsilon_{i j}, \Pi_{i j}$, and $D_{i j}$, are called hereafter convection, production, dissipation, pressure, and diffusion (molecular+turbulent) terms, respectively. For the sake of conciseness, these transport equations are given in tensorial form, in a Cartesian frame. The production term $P_{i j}$ is expressed in cylindrical coordinates in Sec. V C 2 to make the theoretical analysis possible.

\section{Outer layer}

In order to study the influence of rotation, an order of magnitude analysis of the different terms is performed. 


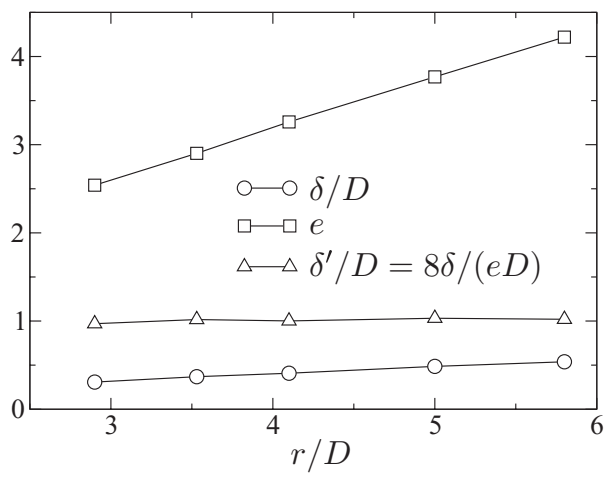

FIG. 10. Radial distribution of the wall jet thickness $\delta$, the entrainment ratio $e$, and the reduced thickness $\delta^{\prime}$. Results given by the EB-RSM.

Since the flow is radial, even if entrainment were negligible and the wall-jet thickness constant, mass conservation would imply a variation as $r^{-1}$ of the radial velocity, such that the scale of change in the $r$-direction, denoted by $L$, must be defined with care in order to correctly characterize the slow evolution of the wall jet due to diffusion. Therefore, the standard definition of $L$ used for plane, almost parallel flows, ${ }^{34} \partial U / \partial x \sim U_{\max } / L$ (where $\sim$ stands for of the order of magnitude of), is replaced by

$$
\frac{1}{r} \frac{\partial r V_{r}}{\partial r} \sim \frac{V_{r \max }}{L}
$$

This definition satisfies two desirable features: first, the mass conservation equation (10) shows that the order of magnitude of the wall-normal velocity $V_{z}$ can be expressed under the same form as in the case of plane flows,

$$
V_{z} \sim \frac{\delta}{L} V_{r \max }
$$

where $\delta / L \ll 1$; second, a rigorous definition of the scale $L$ is possible, since Eq. (9) yields

$$
\frac{1}{r} \frac{\partial r V_{r \max }}{\partial r}=-V_{r \max } \frac{1}{\delta^{\prime}} \frac{\partial \delta^{\prime}}{\partial r}
$$

such that

$$
\frac{1}{L}=\frac{1}{\delta^{\prime}} \frac{\partial \delta^{\prime}}{\partial r} .
$$

It will be shown below that self-similarity implies a linear variation of the radial length scale $L$, compatible with power laws for $\delta / D, e$, and $\delta^{\prime} / D .^{28}$ The least square method applied to the results shown in Fig. 10, gives exponents about $0.80,0.74$, and 0.06 for $\delta / D, e$, and $\delta^{\prime} / D$, respectively. Owing to the definition (19), if $\delta^{\prime} / D$ varies like $(r / D)^{\beta}$, the length scale $L$ satisfies

$$
L=\frac{r}{\beta},
$$

such that $r / L \approx 0.06$ can also be considered small compared to unity.

Obtaining the following orders of magnitude is then straightforward:

$$
\begin{gathered}
\frac{\partial V_{r}}{\partial r}=\frac{1}{r} \frac{\partial}{\partial r}\left(r V_{r}\right)-\frac{V_{r}}{r} \sim \frac{V_{r \max }}{r}\left(\frac{r}{L}+1\right) \sim \frac{V_{r \max }}{r}, \\
\frac{\partial V_{z}}{\partial z}=-\frac{1}{r} \frac{\partial}{\partial r}\left(r V_{r}\right) \sim \frac{V_{r \max }}{L} .
\end{gathered}
$$


Since the viscous terms are negligible, the outer layer of the wall jet behaves as a free jet, i.e., the wall-normal momentum budget (13) becomes

$$
\underbrace{V_{r} \frac{\partial V_{z}}{\partial r}}_{\sim \frac{V_{r \max }^{2} \delta}{L^{2}}}+\underbrace{V_{z} \frac{\partial V_{z}}{\partial z}}_{\sim \frac{V_{r \max }^{2}}{L^{2}}}=-\frac{1}{\rho} \frac{\partial P}{\partial z}-\underbrace{\frac{\partial \overline{v_{r} v_{z}}}{\partial r}}_{\sim \frac{u^{2}}{r}}-\underbrace{\frac{\overline{v_{r} v_{z}}}{r}}_{\sim \frac{u^{2}}{r}}-\underbrace{\frac{\partial \overline{v_{z}^{2}}}{\partial z}}_{\sim \frac{u^{2}}{\delta}},
$$

where $u$ denotes the order of magnitude of the velocity fluctuations. Unless $u^{2} / V_{r \max }^{2}$ is as small as $\delta^{2} / L^{2}$, this equation reduces, at leading order, to

$$
\frac{1}{\rho} \frac{\partial P}{\partial z}+\frac{\partial \overline{v_{z}^{2}}}{\partial z}=0 .
$$

Integrating along the wall-normal direction shows that $P / \rho+\overline{v_{z}^{2}}$ is a constant and taking the derivative in the radial direction yields

$$
\frac{1}{\rho} \frac{\partial P}{\partial r}=-\frac{\partial \overline{v_{z}^{2}}}{\partial r} \sim \frac{u^{2}}{r},
$$

such that the radial momentum budget (11) reads

$$
\underbrace{V_{r} \frac{\partial V_{r}}{\partial r}}_{\sim \frac{V_{r \max }^{2}}{r}}+\underbrace{V_{z} \frac{\partial V_{r}}{\partial z}}_{\sim \frac{V_{r \max }^{2}}{L}}=\underbrace{\frac{\partial \overline{v_{z}^{2}}}{\partial r}}_{\sim \frac{u^{2}}{r}}-\underbrace{\frac{\partial \overline{v_{r}^{2}}}{\partial r}}_{\sim \frac{u^{2}}{r}}-\underbrace{\frac{\overline{v_{r}^{2}}}{r}}_{\sim \frac{u^{2}}{r}}-\underbrace{\frac{\partial \overline{v_{r} v_{z}}}{\partial z}}_{\sim \frac{u^{2}}{\delta}}+\underbrace{\frac{\overline{v_{\theta}^{2}}}{r}}_{\sim \frac{u^{2}}{r}},
$$

and, consequently, since $V_{r \max }^{2} / r$ and $u^{2} / \delta$ are dominant, we have

$$
\frac{u^{2}}{V_{r \max }^{2}} \sim \frac{\delta}{r}
$$

which is indeed large compared to $\delta^{2} / L^{2}$.

Similarly, the energy conservation equation (14) becomes

$$
\underbrace{V_{r} \frac{\partial T}{\partial r}}_{\sim \frac{V_{r \max } \Delta T}{L}}+\underbrace{V_{z} \frac{\partial T}{\partial z}}_{\sim \frac{V_{r \max } \Delta T}{L}}=-\underbrace{\frac{1}{r} \frac{\partial}{\partial r}\left(r \overline{v_{r} \vartheta}\right)}_{\sim \frac{u \Theta}{L}}-\underbrace{\frac{\partial \overline{v_{z} \vartheta}}{\partial z}}_{\sim \frac{u \Theta}{\delta}},
$$

where $\Delta T=T_{w}-T_{\infty}$ is the difference between wall and ambient temperatures, and $\Theta$ stands for the order of magnitude of the temperature fluctuations. Since the third term in Eq. (28) is negligible, using Eq. (27) to eliminate $V_{r \max }$ yields

$$
\Theta \sim \frac{r^{1 / 2} \delta^{1 / 2}}{L} \Delta T .
$$

Therefore, keeping only the dominant terms, the wall jet obeys the simplified conservation equations

$$
\begin{aligned}
\frac{1}{r} \frac{\partial}{\partial r}\left(r V_{r}\right)+\frac{\partial V_{z}}{\partial z} & =0 \\
V_{r} \frac{\partial V_{r}}{\partial r}+V_{z} \frac{\partial V_{r}}{\partial z} & =-\frac{\partial \overline{v_{r} v_{z}}}{\partial z} \\
V_{r} \frac{\partial T}{\partial r}+V_{z} \frac{\partial T}{\partial z} & =-\frac{\partial \overline{v_{z} \vartheta}}{\partial z}
\end{aligned}
$$

and the introduction of a self-similar solutions of the form

$$
\begin{gathered}
V_{r}=V_{r \max } F(\xi) ;-\overline{v_{r} v_{z}}=V_{r \max }^{2} f(\xi) ; T-T_{\infty}=\Delta T G(\xi) ; \\
\text { and } \overline{v_{z} \vartheta}=-V_{r \max } \Delta T g(\xi),
\end{gathered}
$$




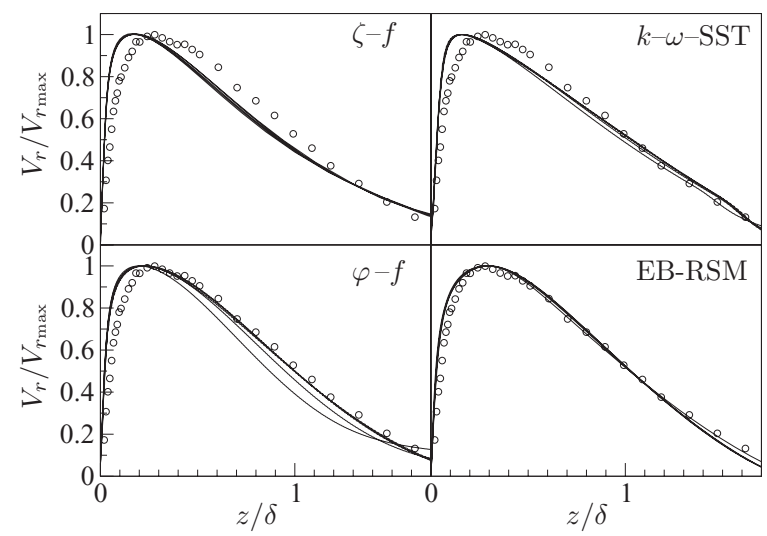

FIG. 11. Self-similarity of the radial velocity profiles. Symbols represent the experimental profile measured at $r=5.80 D$.

where $\xi=z / \delta$, leads to

$$
\frac{\delta}{V_{r \max }} \frac{\partial V_{r \max }}{\partial r} F^{2}-\frac{\partial \delta}{\partial r} \xi F F^{\prime}-\frac{\delta}{L} F^{\prime} \int_{\xi}^{\infty} F \mathrm{~d} \tilde{\xi}-\frac{\partial \delta}{\partial r} F^{\prime} \int_{\xi}^{\infty} \tilde{\xi} F^{\prime} \mathrm{d} \tilde{\xi}=f^{\prime}
$$

and

$$
\frac{\delta}{\Delta T} \frac{\partial \Delta T}{\partial r} F G-\frac{\partial \delta}{\partial r} \xi F G^{\prime}-\frac{\delta}{L} G^{\prime} \int_{\xi}^{\infty} F \mathrm{~d} \tilde{\xi}-\frac{\partial \delta}{\partial r} G^{\prime} \int_{\xi}^{\infty} \tilde{\xi} F^{\prime} \mathrm{d} \tilde{\xi}=g^{\prime} .
$$

A self-similar solution is possible if

$$
a=\frac{\delta}{V_{r \max }} \frac{\partial V_{r \max }}{\partial r}, \quad b=\frac{\partial \delta}{\partial r}, \quad c=\frac{\delta}{L}, \quad \text { and } \quad d=\frac{\delta}{\Delta T} \frac{\partial \Delta T}{\partial r}
$$

have the same $r$-dependency (their ratios are constant).

Figure 11 shows that the $\zeta-f$ and EB-RSM better satisfy the self-similarity hypothesis than the other models, in particular after the location $r=3.53 \mathrm{D}$, and only the EB-RSM model provides a shape of the function $f(\xi)$ close to the experiments.

Moreover, Eqs. (18) and (19) show that

$$
\frac{\partial V_{r \max }}{\partial r}+\frac{V_{r \max }}{r}=-\frac{V_{r \max }}{L},
$$

such that $(a+c) / c=-L / r$. Since this ratio must be constant as stated above, $L$ grows linearly with $r$, the growth rate $\beta^{-1}$ being the reciprocal of the exponent of the power law for $\delta^{\prime}$ (see Eq. (20)). Since

$$
\frac{b}{c}=\frac{L}{\delta} \frac{\partial \delta}{\partial r}
$$

must be a constant, this linear variation $L=\beta^{-1} r$ implies a power law for $\delta$, and consequently, for $e$ $=8 \delta / \delta^{\prime}$, a conclusion that was anticipated above.

Furthermore, Eq. (37) yields

$$
\frac{1}{V_{r \max }} \frac{\partial V_{r \max }}{\partial r}=-\frac{1+\beta}{r}
$$

such that $V_{r \max }$ varies as

$$
\frac{V_{r \max }}{U_{j}} \propto\left(\frac{r}{D}\right)^{-1-\beta}
$$

where $\propto$ stands for proportional to. Moreover, since

$$
\frac{d}{a}=\frac{1}{\Delta T} \frac{\partial \Delta T}{\partial r}\left(\frac{1}{V_{r \max }} \frac{\partial V_{r \max }}{\partial r}\right)^{-1}
$$




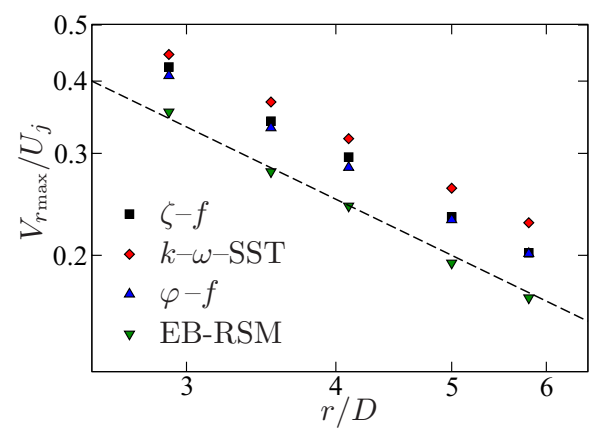

FIG. 12. Radial evolution of the maximum velocity $V_{r \max }$ in $\log -\log$ scale. The dashed line is the curve $V_{r \max } / U_{j}=(r / D)^{-1}$.

must be a constant, $\Delta T$ also varies as a power law of $r / D$.

Finally, as shown in Fig. 10 for the EB-RSM, the reduced thickness $\delta^{\prime} / D$ exhibits a very slow variation in the radial direction, and can be, for order of magnitude evaluations below, considered a constant (i.e., $\beta \ll 1$, and $L \rightarrow \infty$ ), and close to unity. Consequently, Eq. (40) leads to

$$
\frac{V_{r \max }}{U_{j}} \approx\left(\frac{r}{D}\right)^{-1}
$$

It can be seen in Fig. 12 that the maximum velocity approximately varies as $(r / D)^{-1}$ for all the models.

\section{Inner layer}

A complete self-similar solution is possible only if it is assumed that the friction velocity $u_{\tau}$ scales with $V_{r \max }$, i.e., the inner layer is in equilibrium with the outer layer. ${ }^{32}$ This remark also holds for the temperature, i.e., the friction temperature $T_{\tau}$ must scale with $\Delta T$. The boundary layer can be described using Kármán's method, ${ }^{7,8,27}$ i.e., it can be considered to behave as a standard boundary layer without pressure gradient. For instance, assuming a Blasius power law for the velocity profile, the wall friction can be shown to behave as

$$
\frac{\tau_{w}}{\frac{1}{2} \rho V_{r \max }^{2}} \propto\left(\frac{v}{V_{r \max } \delta_{\max }}\right)^{1 / 4},
$$

where $\delta_{\max }$ is the location of the velocity maximum. In a self-similar solution, $\delta_{\max } / \delta$ is a constant, such that $\delta_{\max }$ varies as $(r / D)^{0.8}$ as $\delta$ does. It has been shown in Sec. IV B 2 that $V_{r \max } / U_{j}=(r / D)^{-1}$, such that the friction coefficient based on $U_{j}$ behaves as

$$
C_{f}=\frac{\tau_{w}}{\frac{1}{2} \rho U_{j}^{2}}=\frac{V_{r \max }^{2}}{U_{j}^{2}} \frac{\tau_{w}}{\frac{1}{2} \rho V_{r \max }^{2}} \propto \frac{1}{R e_{j}^{1 / 4}}\left(\frac{r}{D}\right)^{-1.95} .
$$

Figure 13 shows that the results given by the EB-RSM very well satisfy the evolution in $(r / D)^{-1.95}$ after $r / D \approx 2.5$, which supports the self-similarity hypothesis as well as the equilibrium between the inner and outer layers.

As concerns heat transfer, the constant turbulent Prandtl number hypothesis implies ${ }^{35,36}$ that the local Stanton number is related to the friction velocity by

$$
S t=\frac{\dot{q}_{w}}{\rho C_{p} V_{r \max } \Delta T}=\frac{1}{\operatorname{Pr}_{t}\left(1+C \frac{u_{\tau}}{V_{r \max }}\right)} \frac{u_{\tau}^{2}}{V_{r \max }^{2}},
$$

where $C$ is a constant (for details, see, e.g., Arpaci and $\operatorname{Larsen}^{37}$ ). Since $P r_{t} \approx 1$ and $C u_{\tau} / V_{r \max } \ll 1$, the Reynolds analogy $S t \propto u_{\tau}^{2} / V_{r \max }^{2}$ is valid, such that the Nusselt number $N u=\dot{q}_{w} D /(\lambda \Delta T)$ scales 


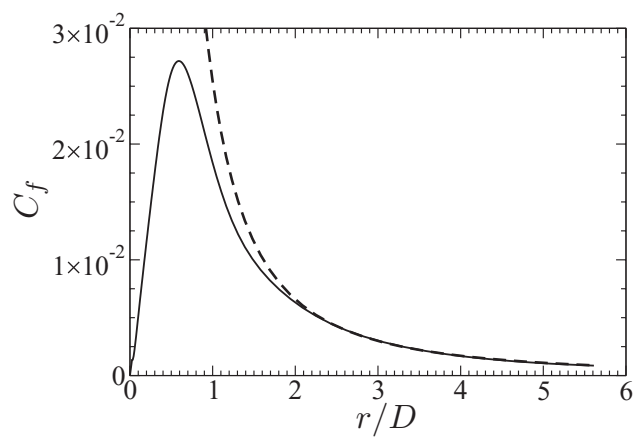

FIG. 13. Friction coefficient given by the EB-RSM. The dashed line is a least-square fit in the region $r / D>2.5$ using Eq. (44).

as

$$
N u=\frac{\rho C_{p}}{\lambda} D V_{r \max } S=\operatorname{Pr} \operatorname{Re}_{j} \frac{V_{r \max }}{U_{j}} S \propto \operatorname{Re}_{j}^{3 / 4} \frac{D}{r} .
$$

This result is obviously compatible with the power-law solution obtained for the outer layer if $\beta$ $\ll 1$ at the end of Sec. IV B 2, which again supports the assumption of inner and outer layers in equilibrium. The behavior $N u \propto(r / D)^{-1}$ is well confirmed by the dotted line drawn in Fig. 4, at least for the experimental and EB-RSM results.

\section{ANALYSIS OF THE INFLUENCE OF ROTATION}

This section is devoted to the analysis of the leading order mechanisms that modify the flow when the disk is rotating, in the wall jet region beyond $r / D>2.5$, since the flow is dominated by the pressure gradient in the impingement region, as shown in Fig. 3.

\section{A. Comparison of the thicknesses}

The case of the boundary layer developing on a rotating disk in a quiescent environment (without the impinging jet) was first considered by von Kármán. ${ }^{7}$ In this case, if Blasius power-law velocity profiles are assumed for both the radial and azimuthal velocity profiles

$$
V_{r}=\beta r \omega\left(\frac{z}{\ell}\right)^{1 / 7}\left(1-\frac{z}{\ell}\right) \quad ; \quad V_{\theta}=r \omega\left[1-\left(\frac{z}{\ell}\right)^{1 / 7}\right],
$$

it can be shown (for details, see, e.g., Dorfman ${ }^{9}$ or Schlichting ${ }^{38}$ ) that the thickness $\ell$ of the boundary layer scales with the local Reynolds number $R e_{r}=r^{2} \omega / \nu$

$$
\frac{\ell}{r} \propto\left(\frac{r^{2} \omega}{v}\right)^{-1 / 5}=\alpha^{-1 / 5} \operatorname{Re}_{j}^{-1 / 5}
$$

where

$$
\alpha=\frac{r^{2} \omega}{U_{j} D}
$$

i.e., decreases with increasing rotation rate and radial location.

For weak rotation rates, orders of magnitude found in Sec. IV B 2 and the self-similar solution (33) still hold, and rotation only moderately affects the thickness $\delta$ of the wall jet. In Fig. 14, although the lowest rotation rate, $\omega D / U_{j}=0.12$, is already too high for the rotational effect to be negligible, it can be observed, for the EB-RSM, that $\delta$ still varies as a power of the radius $\left(\delta / D \propto(r / D)^{\gamma}\right)$. Moreover, Fig. 15 shows that the thickness of the azimuthal boundary layer satisfies Eq. (48), which indicates that it is not strongly affected either by the presence of the wall jet. Thus, the ratio of the azimuthal 


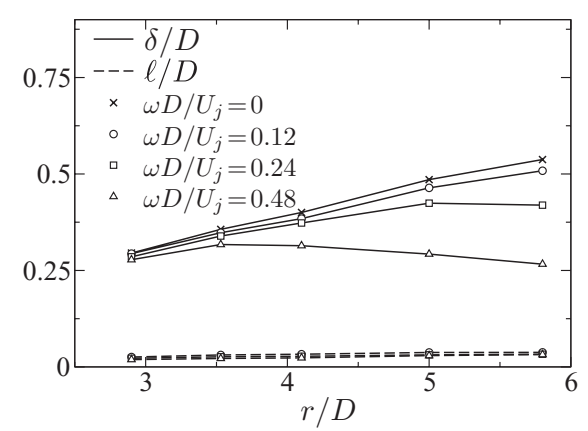

FIG. 14. Comparison of the thicknesses of the wall jet and the azimuthal boundary layer for the four rotation rates $(\ell / D$ is not defined for $\left.\omega D / U_{j}=0\right)$. EB-RSM results.

boundary layer thickness to the wall jet thickness is

$$
\frac{\ell}{\delta} \propto\left(\frac{r^{2} \omega}{v}\right)^{-1 / 5}=\alpha^{-1 / 5} \operatorname{Re}_{j}^{-1 / 5}\left(\frac{r}{D}\right)^{1-\gamma} .
$$

Owing to the high Reynolds number, this ratio is small, as can be seen in Fig. 14, where $\ell$ is obtained from

$$
\ell=\frac{1}{r \omega} \int_{0}^{\infty} V_{\theta} \mathrm{d} z
$$

Since $\ell$ decreases with the rotation rate, the increasing influence of rotation on the outer layer of the wall jet cannot be attributed to a growing of $\ell$. Therefore, the scenario to be retained is an interaction between the rotation-induced, azimuthal boundary layer, and the inner layer of the wall jet, which leads to a modification of the equilibrium, shown in Sec. IV B 3, between the inner and outer layers.

\section{B. Limit of strong rotations}

The modification of the wall jet for strong rotation rates is thus to be related to a modification of the inner layer. In this layer, centrifugal acceleration becomes dominant in the radial momentum equation in the limit $\omega \rightarrow \infty$, leading to

$$
\underbrace{V_{r} \frac{\partial V_{r}}{\partial r}}_{\sim \frac{V_{r}^{2}}{r}}-\underbrace{\frac{V_{\theta}^{2}}{r}}_{\sim r \omega^{2}}=0,
$$

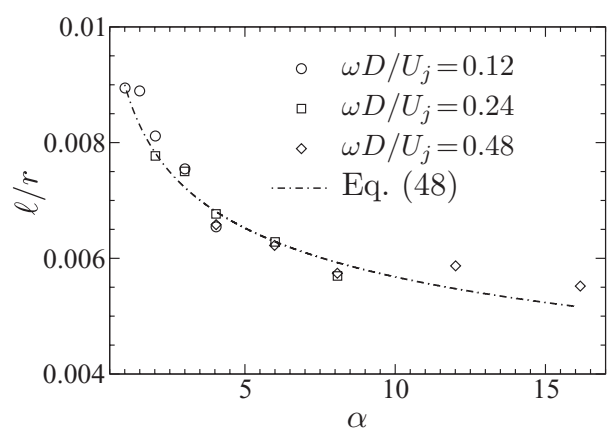

FIG. 15. Ratio of the thickness of the azimuthal boundary layer to the radial location as a function of $\alpha$, for the 3 rotation rates. The dashed line is a least-square fit using Eq. (48). EB-RSM results. 
which yields

$$
V_{r} \sim r \omega .
$$

The primary effect of rotation is thus an acceleration of the inner layer.

Although, as stated above, the centrifugal acceleration does not directly affect the outer layer, the acceleration of the inner layer necessarily leads to a suction of fluid at the bottom of the outer layer. Indeed, integrating the mass conservation equation (10) between the wall and the upper limit of the wall layer $z=\delta_{\max }$ yields

$$
V_{z}=-\int_{0}^{\delta_{\max }} \frac{1}{r} \frac{\partial r V_{r}}{\partial r} \mathrm{~d} z,
$$

such that the radial acceleration generates a negative $V_{z}$ at $z=\delta_{\max }$. Equation (53) shows that the integrand scales as $\omega$, such that $V_{z}$ at $z=\delta_{\max }$ scales as

$$
\delta_{\max } \omega .
$$

Therefore, the acceleration in the wall layer modifies the condition at the lower bound of the outer layer, and, similarly, mass conservation between $z=\delta_{\max }$ and infinity shows that $V_{r}$ in the outer layer also scales as

$$
V_{r} \sim V_{r \max } \sim r \omega .
$$

Moreover, it was shown in Sec. IV, for the case without rotation, that $r V_{r \max }$ is constant and equal to $U_{j} D$, since $\delta^{\prime} \approx D$. The influence of rotation on the wall jet can then be characterized by the modification of $r V_{r \max }$, i.e., by

$$
\frac{r V_{r \max }}{\left(r V_{r \max }\right)_{0}} \sim \frac{r V_{r \max }}{U_{j} D} \sim \alpha,
$$

where the index ${ }_{0}$ denotes the value for the non-rotational case. Since Eq. (9) is exact, this result is equivalent to

$$
\frac{\delta^{\prime}}{\delta_{0}^{\prime}} \sim \frac{1}{\alpha},
$$

i.e., the acceleration of the mean flow in the radial direction implies a thinning of the wall jet, which was observed in Fig. 5.

These two effects are not related to turbulence, such that for strong rotation rates and regions close to the edge $(r=6 D)$ of the disk (i.e., large values of $\alpha$ ), the dynamics of the flow is dominated by centrifugal acceleration, and the influence of the turbulence model is weak as seen in Figs. 3 and 5. Therefore, reproducing the effects of rotation is more challenging for turbulence models in the region located between the impingement region and the rotation-dominated region, and for intermediate rotation rates.

Figure 16 remarkably well confirms the scaling laws (57) and (58), for both the experiments and the computations. However, discrepancies are observed in the slopes predicted by the different models, and the Reynolds stress model is closer to the experiments.

One of the main consequences of the fact that the rotation of the disk does not directly affect the outer layer is that entrainment, due to the turbulence at the edge of the outer layer, can be expected to be independent of the rotation rate. Figure 17 shows that, for both the measurements and the EB-RSM, the turbulence in the outer layer (down to $z / D \approx 0.3$ ) is indeed weakly affected by rotation, contrary to the inner layer. Consequently, as can be seen in Fig. 18, the entrainment ratio is independent of the rotation rate. The situation is different for eddy-viscosity models, because the turbulent shear stress is assumed proportional to the mean shear, which is modified by the acceleration and thinning of the wall jet.

Since the thickness $\delta$ of the jet is related to the entrainment ratio $e$ and the reduced thickness $\delta^{\prime}$ by $\delta=e \delta^{\prime} / 8$, and $e$ is independent of the rotation rate, which is the case for both the experimental 

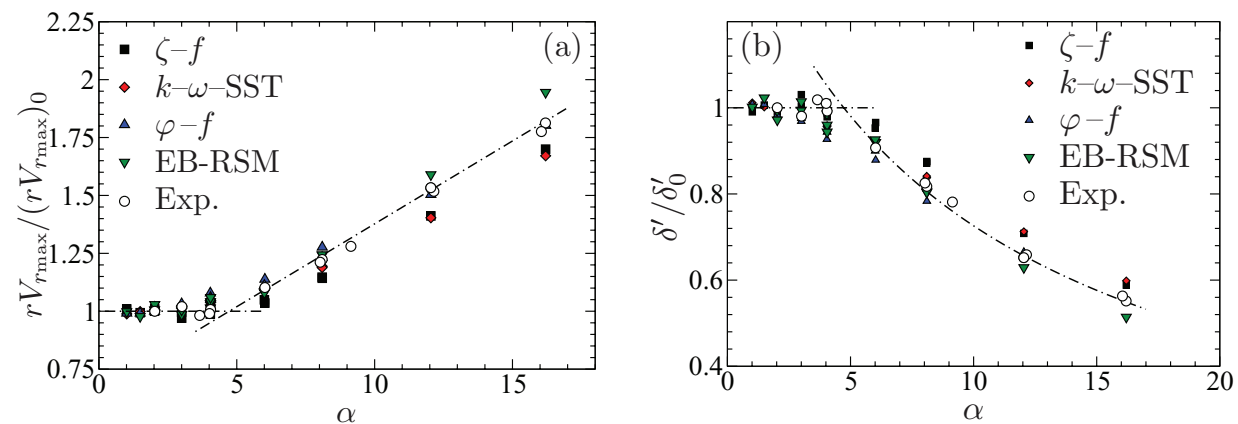

FIG. 16. Scaling with $\alpha$ of (a) $r V_{r \max } /\left(r V_{r \max }\right)_{0}$ and (b) $\delta^{\prime} / \delta_{0}^{\prime}$. The dashed lines represent the theoretical relations fitted to the experimental results in the case of negligible and dominant rotational effects, respectively.
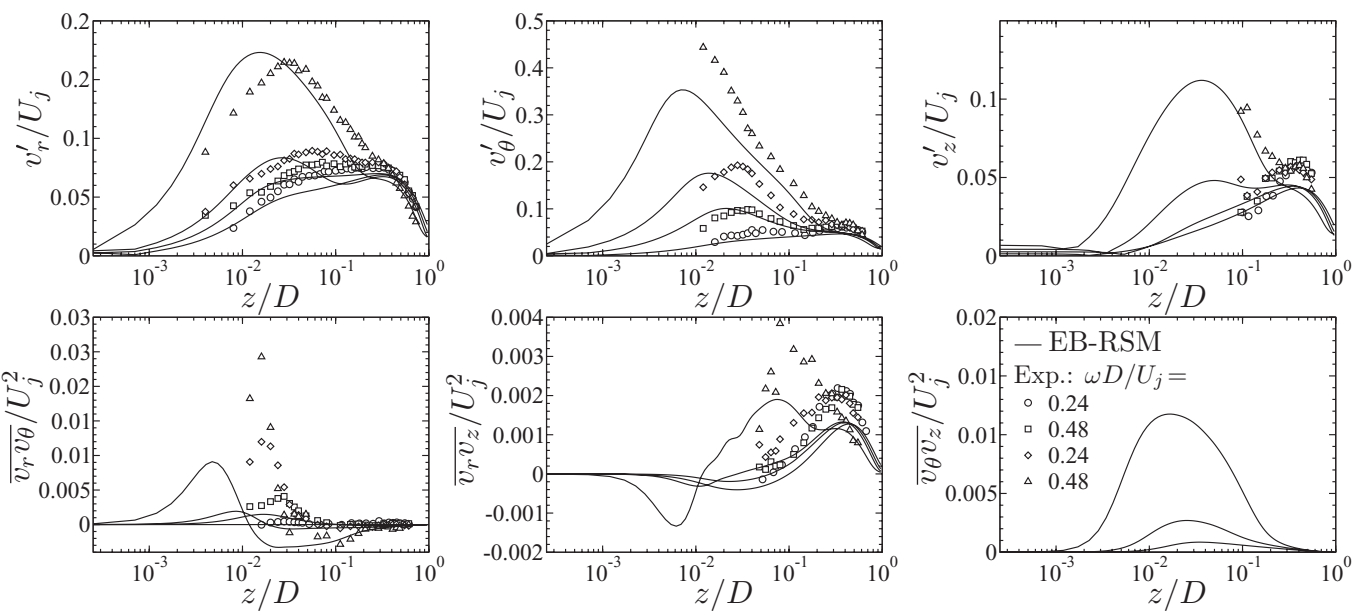

FIG. 17. Influence of rotation on the Reynolds stresses. Comparison of the EB-RSM profiles with the experiments ${ }^{11}$ at $r / D$ $=5.8$.

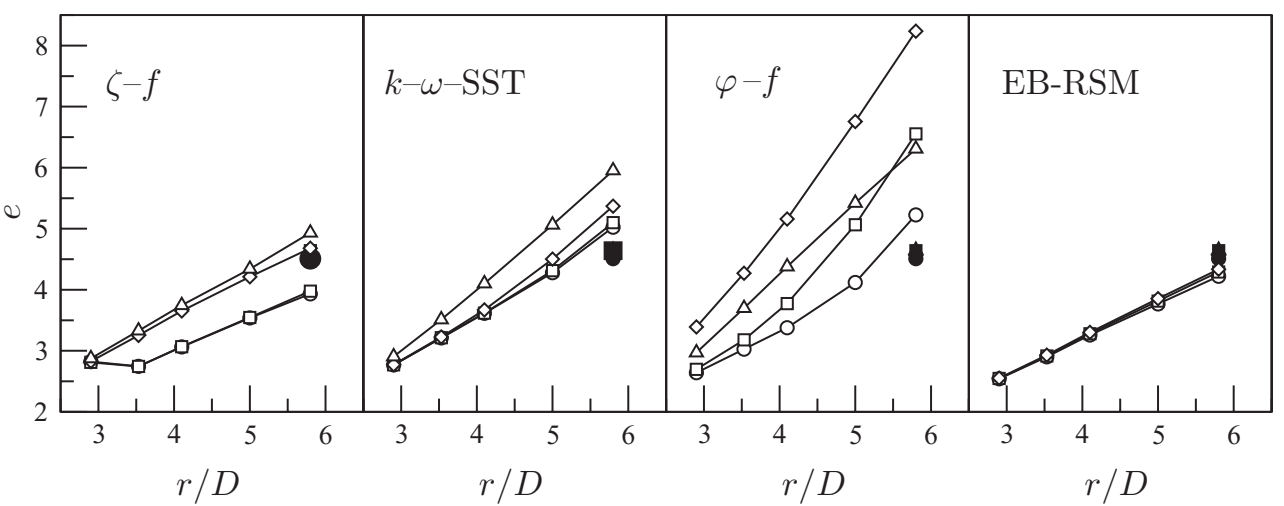

FIG. 18. Radial evolution of entrainment ratio $e$ for the different rotation rates $\omega D / U_{j}: \circ 0.00 ; \square 0.12 ; \triangle 0.24 ; \diamond 0.48$. Filled symbols: Experiments.

and EB-RSM results, we have

$$
\frac{\delta}{\delta_{0}} \sim \alpha^{-1}
$$

Figure 19(a) confirms this scaling for strong rotation rates $(\alpha>5)$. Minagawa and $\mathrm{Obi}^{11}$ conjectured this scaling, but due to limitations inherent to the experimental approach, they monitored the variation 

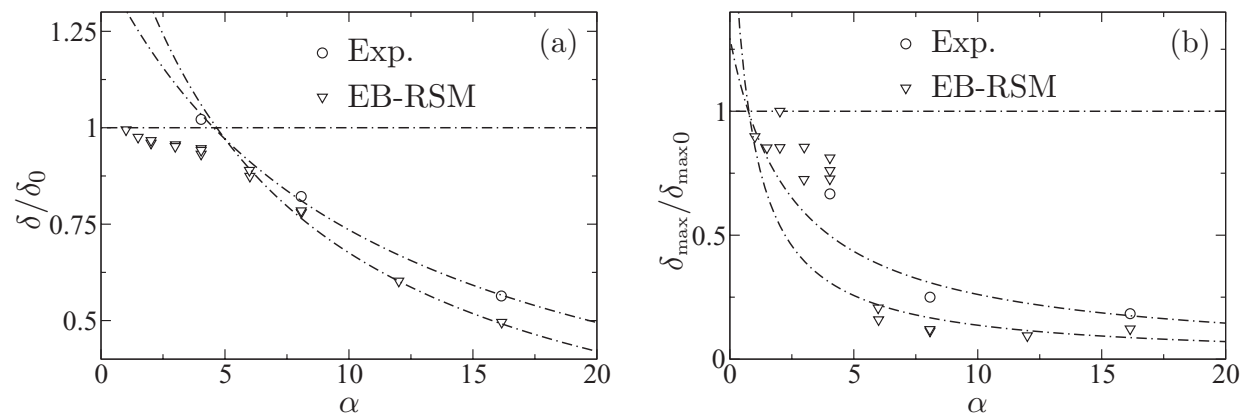

FIG. 19. Scaling with $\alpha$ of (a) $\delta / \delta_{0}$ and (b) $\delta_{\max } / \delta_{\max 0}$. Dashed lines show the theoretical relations for weak rotations (horizontal lines) and for strong rotations $\left(\delta / \delta_{0} \sim \alpha^{-1}\right)$, fitted to the experimental and numerical results.

of the location of the velocity maximum, $\delta_{\max } / \delta_{\max 0}$, and, as shown in Fig. 19(b), this quantity does not scale very well with $\alpha^{-1}$. In case of rotation, the deformation of the velocity profiles breaks the self-similarity, such that the thicknesses $\delta$ and $\delta_{\max }$ are not proportional to each other, and the analysis above shows that $\delta$ is the correct quantity to be used for the investigation of the scaling properties of the rotating wall jet.

As shown in Fig. 6, when the rotation rate is increased, the models are able to reproduce the increase of the Nusselt number that was observed at higher Reynolds numbers by Popiel and Boguslawski. ${ }^{6}$ Figure 20 shows the evolution of the Nusselt number based on the radial location $\mathrm{Nu}_{r}$ $=r N u / D$ as a function of the rotational Reynolds number $R e_{r}=\omega r^{2} / \nu=\alpha R e_{j}$. The values have been extracted at several radial locations for the 4 rotation rates. It can be observed that the results of the EB-RSM, $\zeta-f$ and $k-\omega-$ SST models well satisfy the scaling proposed by Popiel and Boguslawski, ${ }^{6}$ $N u_{r}=f\left(R e_{r}\right)$, contrary to the $\varphi-f$ model that shows some scatter, due to the lack of self-similarity of the solution, as seen in Fig. 11. For high values of $R e_{r}$, it is expected that the Nusselt number approaches the asymptote $N u_{r} \propto R e_{r}^{0.8}$ applicable to a rotating disk in still air. ${ }^{9}$ Despite the fact that the rotation rate is not sufficiently high, it can be seen that the present computations, for all the models, seem to approach the correct asymptotic behavior.

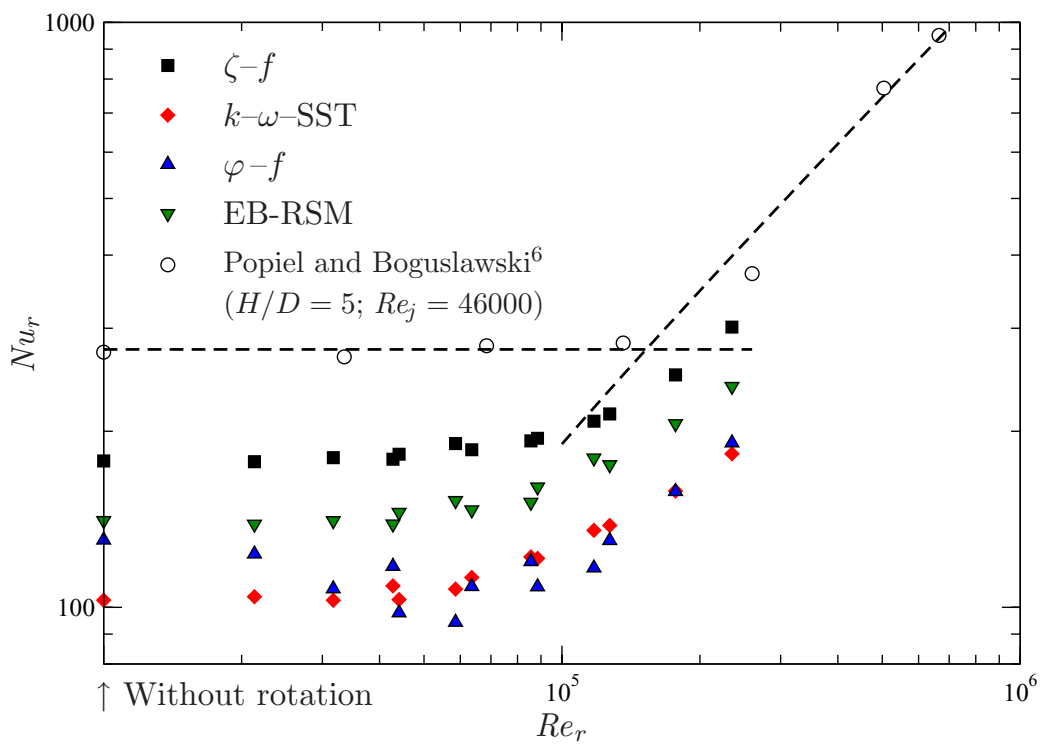

FIG. 20. Nusselt number $N u_{r}=r N u / D$ as a function of $R e_{r}$. The dashed lines represent least-square fits of the experiments using a constant $N u_{r}$ for weak rotation and $N u_{r} \propto R e_{r}^{0.8}$ for strong rotation (correlation for a disk rotating in still air, ${ }^{99}$ ). The values without rotation are artificially plotted on the ordinate axis. 


\section{Analysis of the appearance of rotational effects}

Sections IV B and V B focused on the behavior of the wall jet in cases without rotation and dominated by rotation, respectively. Section V C is devoted to the analysis of intermediate rotation rates, aiming at identifying a scenario describing the transition from one situation to the other, and defining criteria to anticipate the appearance or not of significant rotational effects.

\section{Momentum equation}

As long as the rotation rate is sufficiently weak, the self-similar solution for the outer layer holds, and the description of the inner layer given in Sec. IV B 3 is valid. In the inner layer, the radial velocity and length scales are $u_{\tau}$ and $v / u_{\tau}$, respectively, where, following Eq. (44):

$$
u_{\tau} \sim \frac{1}{R e_{j}^{1 / 8}} \frac{U_{j} D}{r}\left(\frac{r}{D}\right)^{0.025} \sim \frac{1}{R e_{j}^{1 / 8}} \frac{U_{j} D}{r} .
$$

For the evaluation of the orders of magnitude of the different terms in the equations, the factor $(r / D)^{0.025}$ can be considered unity, since it remains smaller than 2 as long as $r / D$ is smaller than $10^{12}$, such that the second approximation in Eq. (60) is used.

Since $V_{\theta} \sim r \omega$, the radial momentum equation reads, at leading order,

$$
\underbrace{-\frac{V_{\theta}^{2}}{r}}_{\sim r \omega^{2}}=\underbrace{\nu \frac{\partial^{2} V_{r}}{\partial z^{2}}}_{\sim R e_{j}^{5 / 8} \frac{U_{j}^{2} D^{2}}{r^{3}}}-\underbrace{\frac{\partial \overline{v_{r} v_{z}}}{\partial z}}_{\sim R e_{j}^{5 / 8} \frac{U_{j}^{2} D^{2}}{r^{3}}} .
$$

Consequently, the relative weight of the centrifugal acceleration in this equation is

$$
\frac{r^{4} \omega^{2}}{R e_{j}^{5 / 8} U_{j}^{2} D^{2}}=\frac{\alpha^{2}}{R e_{j}^{5 / 8}},
$$

such that it remains negligible for $\alpha \ll R e_{j}^{5 / 16}$. In Sec. V B, it was shown, in particular in Figs. 16 and 19, using experimental and numerical results, that the transition to the flow dominated by centrifugal acceleration occurred in the region $\alpha \approx 5$, which suggests the threshold $\alpha \approx 0.25 \operatorname{Re}_{j}^{5 / 16}$.

\section{Reynolds stress transport equation}

Wall rotation does not only affect the mean field, i.e., the momentum equation, but also the turbulence, i.e., the Reynolds stress budget (15). Indeed, the appearance of the azimuthal boundary layer results in a non-zero gradient of the azimuthal velocity, which in turn has a direct influence on the production terms of the Reynolds stress transport equations that explicitly involve this gradient. The modification of these terms can be analytically investigated and quantified, as shown below. Analyzing the behavior of the production term as a function of the rotation rate is of primary importance in order to understand the way the turbulent anisotropy is modified. Other terms in the Reynolds stress budget are indirectly influenced by rotation: the modification of these terms can be seen as a response to the change of the production tensor.

The objective of the subsequent analysis is threefold:

(i) Understanding how rotation induced terms in the production tensor affect the dynamics of turbulence. It can be shown that the energy transfer mechanisms are completely different in the outer and inner layers, as suggested by the results of Secs. V A and V B.

(ii) Identifying the reasons for the relative success of eddy-viscosity models in the prediction of the mean flow, although these models are not built to reproduce such complex energy transfer mechanisms. As will be shown, this success is limited to some flow regimes, i.e., confined to some flow regions and limited to a range of rotation rates, in which the influence of the rotation-induced turbulent anisotropy is negligible. 
(iii) Quantifying criteria that can be used to define these flow regimes, as functions of the flow parameters, namely, the jet bulk velocity and the disk rotation rate.

In the absence of direct numerical simulation results, an a priori evaluation of the terms in the Reynolds stress budgets is not possible. However, an order of magnitude analysis of the turbulent production term can isolate the dominant processes of energy transfer from the mean field to the turbulence. For the remaining terms, numerical solution of the modeled Reynolds stress equations provides an estimate of the influence of rotation on these terms.

The production terms of the components of the Reynolds stress (see Eq. (15)) are significantly modified by rotation, since many terms are zero when $V_{\theta}=0$. Indeed, for the non-rotating case, the components of the production tensor reduce to

$$
\begin{gathered}
P_{r r}=P_{r r}^{(1)}+P_{r r}^{(2)}=-2 \overline{v_{r}^{2}} \frac{\partial V_{r}}{\partial r}-\underline{2 \overline{v_{r} v_{z}} \frac{\partial V_{r}}{\partial z}}, \\
P_{\theta \theta}=P_{\theta \theta}^{(1)}=-2 \overline{v_{\theta}^{2}} \frac{V_{r}}{r}, \\
P_{z z}=P_{z z}^{(1)}+P_{z z}^{(2)}=-2 \overline{v_{r} v_{z}} \frac{\partial V_{z}}{\partial r}-\underline{2 \overline{v_{z}^{2}} \frac{\partial V_{z}}{\partial z},} \\
P_{r z}=P_{r z}^{(1)}+P_{r z}^{(2)}+P_{r z}^{(3)}=-\overline{v_{r}^{2}} \frac{\partial V_{z}}{\partial r}+\overline{v_{r} v_{z}} \frac{V_{r}}{r}-\underline{\overline{v_{z}^{2}} \frac{\partial V_{r}}{\partial z},} \\
P_{r \theta}=P_{\theta z}=0,
\end{gathered}
$$

(the underlined terms are those dominant in the production tensor in the absence of rotation, as will be demonstrated in Sec. V C 2). When the disk is rotated, additional terms appear in the production tensor, denoted by the exponent $(r)$, due to the appearance of nonzero $\overline{v_{r} v_{\theta}}, \overline{v_{\theta} v_{z}}$, and $V_{\theta}$,

$$
\begin{gathered}
P_{r r}^{(r)}=2 \overline{v_{r} v_{\theta}} \frac{V_{\theta}}{r}, \\
P_{\theta \theta}^{(r)}=P_{\theta \theta}^{(r 1)}+P_{\theta \theta}^{(r 2)}=-2 \overline{v_{r} v_{\theta}} \frac{\partial V_{\theta}}{\partial r}-2 \overline{v_{\theta} v_{z}} \frac{\partial V_{\theta}}{\partial z}, \\
P_{r \theta}^{(r)}=P_{r \theta}^{(r 1)}+P_{r \theta}^{(r 2)}+P_{r \theta}^{(r 3)}+P_{r \theta}^{(r 4)}+P_{r \theta}^{(r 5)} \\
=-\overline{v_{r}^{2}} \frac{\partial V_{\theta}}{\partial r}+\overline{v_{r} v_{\theta}} \frac{\partial V_{z}}{\partial z}-\overline{v_{r} v_{z}} \frac{\partial V_{\theta}}{\partial z}+\overline{v_{\theta}^{2}} \frac{V_{\theta}}{r}-\overline{v_{\theta} v_{z}} \frac{\partial V_{r}}{\partial z}, \\
P_{\theta z}^{(r)}=P_{\theta z}^{(r 1)}+P_{\theta z}^{(r 2)}+P_{\theta z}^{(r 3)}+P_{\theta z}^{(r 4)} \\
=-\overline{v_{r} v_{\theta}} \frac{\partial V_{z}}{\partial r}+\overline{v_{\theta} v_{z}} \frac{\partial V_{r}}{\partial r}-\overline{v_{r} v_{z}} \frac{\partial V_{\theta}}{\partial r}-\overline{v_{z}^{2}} \frac{\partial V_{\theta}}{\partial z}, \\
P_{r z}^{(r)}=\overline{v_{\theta} v_{z}} \frac{V_{\theta}}{r} .
\end{gathered}
$$

All the production terms, evaluated from the EB-RSM computations, are plotted in Fig. 21, for the non-rotating case (column 0 , i.e., plots ( $\mathrm{x} 0$ ), where $\mathrm{x}$ varies from (a) to (f)), and for the rotating cases (columns 1,2, and 3, in which the plots are denoted by (x1), (x2), and (x3) for rotation rates $\omega D / U_{j}=0.12,0.24$, and 0.48 , respectively). The scale of the ordinate axis is the same for all the plots to make comparison easier, except for the cases when the variations with rotation makes a rescaling necessary (in this case, the labels are in bold font). The orders of magnitude of the different terms can be readily deduced from Secs. IV B and V C 1, and depend on the region of the flow. 

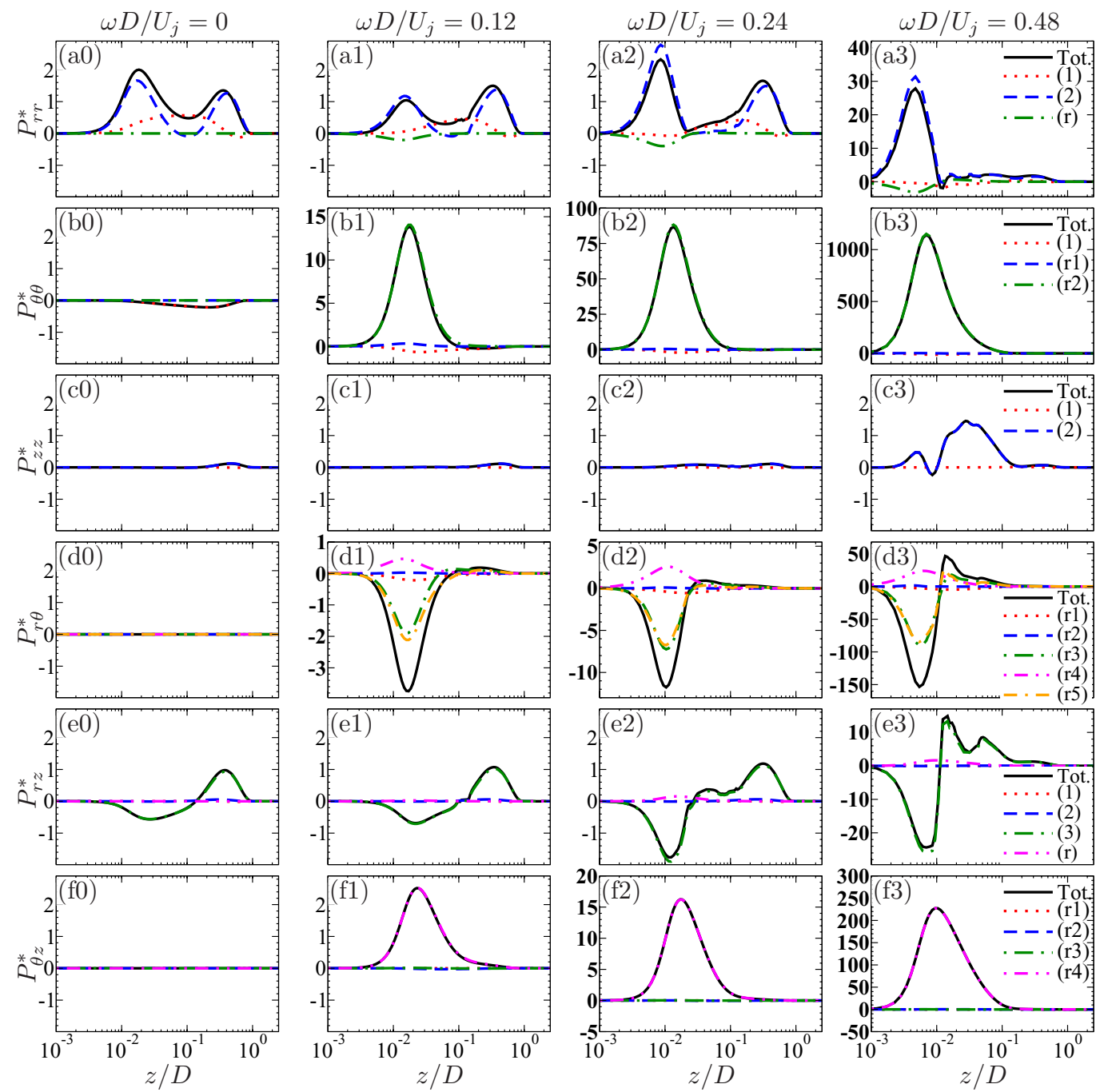

FIG. 21. (a0)-(f3) Profiles of the production terms decomposed into their different contributions taken at the edge of the disk for the four rotation rates, extracted from the EB-RSM computations, made non-dimensional by $U_{j}$ and $D\left(P_{i j}^{*}=P_{i j} D / U_{j}^{3}\right)$ and multiplied by $10^{3}$ for the sake of clarity.

a. Outer layer. From Sec. V A, the conclusion was drawn that the outer layer is not directly affected by the azimuthal boundary layer, which implies that the turbulent field and, in particular, the production term in the outer layer must keep the same structure as in the case without rotation. For this case, using the results of Sec. IV B 2, the following orders of magnitude are obtained:

$$
\begin{gathered}
P_{r r}=P_{r r}^{(1)}+P_{r r}^{(2)} \sim P \frac{\delta}{r}+P \sim P \sim u^{2} \frac{V_{r \max }}{\delta}, \\
P_{\theta \theta}=P_{\theta \theta}^{(1)} \sim P \frac{\delta}{r} \ll P, \\
P_{z z}=P_{z z}^{(1)}+P_{z z}^{(2)} \sim P \frac{\delta^{2}}{L^{2}}+P \frac{\delta}{L} \sim P \frac{\delta}{L} \ll P, \\
P_{r z}=P_{r z}^{(1)}+P_{r z}^{(2)}+P_{r z}^{(3)}=P \frac{\delta^{2}}{L^{2}}+P \frac{\delta}{r}+P \sim P,
\end{gathered}
$$



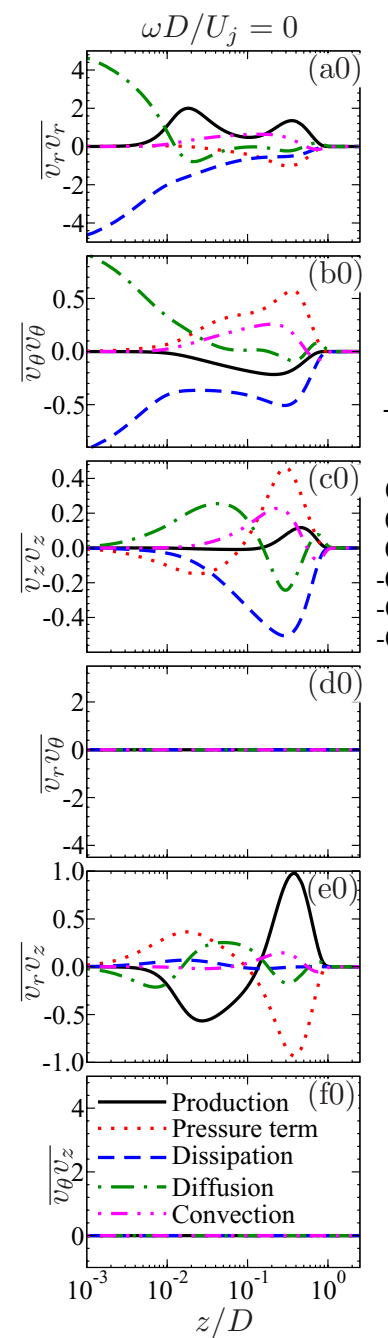
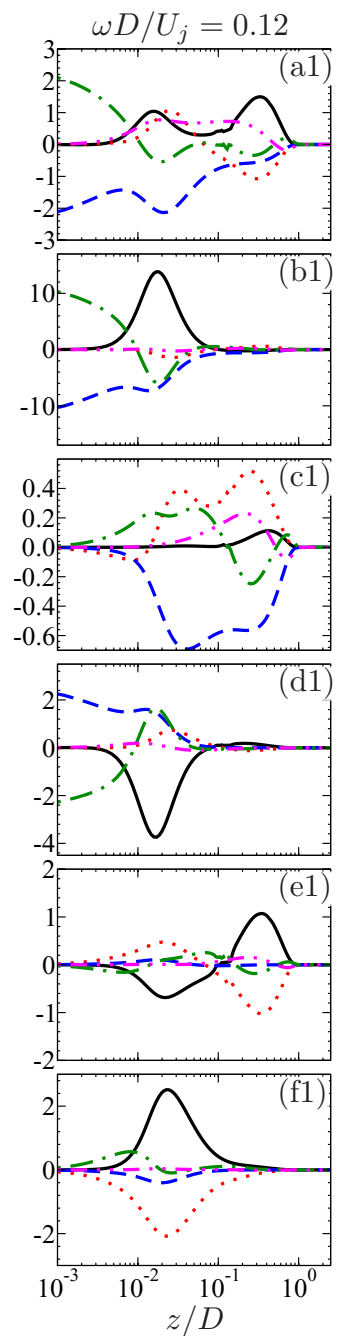
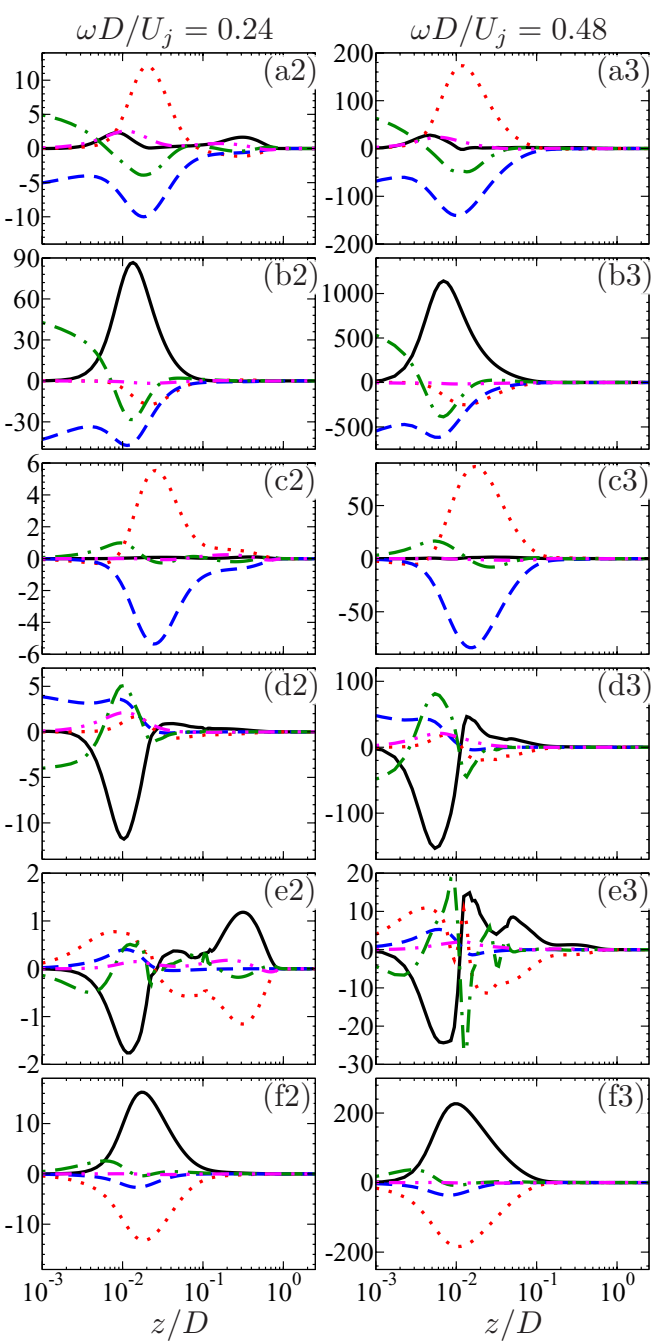

FIG. 22. (a0)-(f3) Profiles of the terms of the budgets of the Reynolds stresses (15) taken at the edge of the disk for the four rotation rates, extracted from the EB-RSM computations. All the terms are made non-dimensional by $U_{j}$ and $D$ and multiplied by $10^{3}$. The legend is in the plot (f0).

where $P$ stands for the turbulent kinetic energy production $\left(P \sim \frac{1}{2} P_{r r}\right.$ here). The outer region can be identified in Fig. 21 by the second peak in $P_{r r}$ (Fig. 21(a0)), approximately located between $z / D=$ 0.1 and $z / D=1$. In this region, the analysis and the computations show that turbulent kinetic energy production is dominated by $P_{r r}^{(2)}$, i.e., the term due to the main velocity gradient, $\partial V_{r} / \partial z$. The other terms, $P_{r r}^{(1)}, P_{\theta \theta}^{(1)}, P_{z z}^{(1)}$, and $P_{z z}^{(2)}$, related to the radial expansion of the jet, are lower at least by a factor of $\delta / r$. Similarly, the dominant term in $P_{r z}$ is $P_{r z}^{(3)}$, the term due to $\partial V_{r} / \partial z$.

It clearly appears in Fig. 21, in particular in columns 1 and 2, that in the outer layer, this description of the production tensor remains valid for all the rotation rates, which confirms the conclusion drawn in Sec. V B that the outer layer is not directly affected by the rotation-induced boundary layer. The production terms are only slightly increased, as can be seen by comparing in Fig. 21 plots (a1) and (a2) to plot (a0), as well as plots (e1) and (e2) to plot (e0), which is simply the consequence of the modification of the velocity gradient $\partial V_{r} / \partial z$, due to the suction of the outer layer by the inner layer. Consequently, the mechanisms driving the evolution of the Reynolds stresses are very similar to the case of a standard boundary layer. Figure 22 shows, for the EB-RSM model, the different terms of the budgets of the Reynolds stresses (15) at the edge of the disk, for the 4 rotation rates, organized in a way similar to Fig. 21. It can be seen in plots (a0), (b0), and (c0) that, as 
in a boundary layer, the velocity gradient $\partial V_{r} / \partial z$ induces turbulent production on the longitudinal component (here, $\overline{v_{r} v_{r}}$ ), and energy is then redistributed to the $\overline{v_{\theta} v_{\theta}}$ and $\overline{v_{z} v_{z}}$ components, via the pressure term (velocity-pressure gradient correlation). A notable difference compared with a standard boundary layer lies in the fact that, due to the radial expansion of the jet, convection is active, for all the components, and, as discussed above, production terms $P_{\theta \theta}$ and $P_{z z}$ are not zero. It is worth pointing out that convection can actually become dominant, for instance, in the $\overline{v_{r} v_{r}}$ budget (plots $(\mathrm{a} 0)$ and (a1)), in the region located around $z / D=0.1$, where the main velocity gradient $\partial V_{r} / \partial z$ is zero, such that production reaches a local minimum (i.e., in the intermediate layer defined by Barenblatt et $a l .{ }^{33}$ ). When the disk rotates (columns 1-3), these energy transfer processes are not significantly modified in the outer layer (the picture will be completely different in the inner layer, as shown below).

b. Inner layer. As can be seen, for instance, in Fig. 21(a0), this region can be identified by the production peak located between the wall and $z / D \approx 0.1$. In this region, as mentioned in Sec. V C 1 , the velocity and length scales are $u_{\tau}$ and $v / u_{\tau}$, respectively, with $u_{\tau}$ given by Eq. (60), such that the different terms involved in the evaluation of the production tensor are of the orders of magnitude

$$
\begin{gathered}
V_{r} \sim \frac{1}{\operatorname{Re}_{j}^{1 / 8}} \frac{U_{j} D}{r}, \quad \frac{\partial V_{r}}{\partial r} \sim \frac{1}{\operatorname{Re}_{j}^{1 / 8}} \frac{U_{j} D}{r^{2}}, \quad \frac{\partial V_{r}}{\partial z} \sim \frac{u_{\tau}^{2}}{v} \sim \operatorname{Re}_{j}^{3 / 4} \frac{U_{j} D}{r^{2}}, \\
\frac{\partial V_{z}}{\partial z} \sim \frac{1}{r} \frac{\partial r V_{r}}{\partial r} \sim \frac{1}{R e_{j}^{1 / 8}} \frac{U_{j} D}{r L}, \quad V_{z} \sim \frac{\partial V_{z}}{\partial z} \frac{v}{u_{\tau}} \sim \frac{1}{R e_{j}} \frac{U_{j} D}{L}, \quad u^{2} \sim u_{\tau}^{2} \sim \frac{1}{R e_{j}^{1 / 4}} \frac{U_{j}^{2} D^{2}}{r^{2}} .
\end{gathered}
$$

The evaluation of the orders of magnitude of the production terms is then straightforward

$$
\begin{gathered}
P_{r r}=P_{r r}^{(1)}+P_{r r}^{(2)} \sim \frac{1}{R e_{j}^{7 / 8}} P+P \sim P \sim R e_{j}^{1 / 2} \frac{U_{j}^{3} D^{3}}{r^{4}}, \\
P_{\theta \theta} \sim \frac{1}{R e_{j}^{7 / 8}} P \ll P, \\
P_{z z}=P_{z z}^{(1)}+P_{z z}^{(2)} \sim \frac{1}{R e_{j}^{7 / 4}} \frac{r^{2}}{L^{2}} P+\frac{1}{R e_{j}^{7 / 8}} \frac{r}{L} P \sim \frac{1}{R e_{j}^{7 / 8}} \frac{r}{L} P \ll P, \\
P_{r z}=P_{r z}^{(1)}+P_{r z}^{(2)}+P_{r z}^{(3)}=\frac{1}{R e_{j}^{7 / 4}} \frac{r^{2}}{L^{2}} P+\frac{1}{R e_{j}^{7 / 8}} P+P \sim P .
\end{gathered}
$$

These results, confirmed by the computational profiles shown in Fig. 21, show that the dominant terms are the same as for the outer layer, i.e., $P_{r r}^{(2)}$ in the turbulent kinetic energy production (plots (a0), (b0), and (c0)) and $P_{r z}^{(3)}$ in $P_{r z}($ plot (e0)).

The major difference between the inner and the outer layers is that the former is, contrary to the latter, directly affected by rotation, such that additional terms appear in the production tensor. The influence of these terms increases with the rotation rate (see Fig. 21, columns 1-3), and, for the purpose of identifying a threshold, the analysis below considers the introduction in the equations of a gradually increasing rotation rate.

As long as the rotation rate is sufficiently weak, the production terms due to rotation are negligible compared to those already present in the non-rotating case, such that Eqs. (77) to (82) still hold. However, since $P_{r \theta}=P_{\theta z}=0$, the production terms $P_{r \theta}^{(r)}$ and $P_{\theta z}^{(r)}$ are of leading order, even for weak rotation rates (see Figs. 21(d1) and 21(f1)). As concerns $P_{r \theta}^{(r)}$, the order of magnitude 
analysis leads to

$$
P_{r \theta}^{(r)}=\underbrace{P_{r \theta}^{(r 1)}}_{\sim u_{\tau}^{2} \omega}+\underbrace{P_{r \theta}^{(r 2)}}_{\sim \frac{u_{\tau}^{2}}{R e_{j}^{7 / 8}} \frac{r^{2}}{\ell L}}+\underbrace{P_{r \theta}^{(r 3)}}_{\sim u_{\tau}^{2} \omega_{\bar{\ell}}^{\frac{r}{\ell}}}+\underbrace{P_{r \theta}^{(r 4)}}_{\sim u_{\tau}^{2} \omega}+\underbrace{P_{r \theta}^{(r 5)}}_{\sim u_{\tau}^{2} \omega_{\frac{r}{\ell}}} \sim P_{r \theta}^{(r 3)}+P_{r \theta}^{(r 5)} \sim \alpha \frac{U_{j} D}{r \ell} u_{\tau}^{2} .
$$

In this analysis, $\overline{v_{r} v_{\theta}} \sim \overline{v_{\theta} v_{z}} \sim\left(\alpha r u_{\tau}^{2}\right) /\left(R e_{j}^{3 / 4} \ell\right)$ has been used. The straightforward but long evaluation of the orders of magnitude of these Reynolds stresses is given in the Appendix. Equation (83) is well corroborated by profiles extracted from the EB-RSM computations shown in Figs. 21(d1)-21(d3): it can be observed that the two terms $P_{r \theta}^{(r 3)}$ and $P_{r \theta}^{(r 5)}$ are almost equal and each represent roughly $50 \%$ of the total in the inner layer. However, the term $P_{r \theta}^{(r 4)}$ is not negligible when the rotation rate is not sufficiently weak, because turbulence gradually departs from the hypothesis $\overline{v_{\theta}^{2}} \sim \overline{v_{r}^{2}} \sim u^{2}$, due to the strong velocity gradient $\partial V_{\theta} / \partial z$ that produces a component $\overline{v_{\theta}^{2}}$ much higher than the other Reynolds stresses, as shown in Fig. 17.

In $P_{\theta z}^{(r)}$, a single term, $P_{\theta z}^{(r 4)}$, provides the dominant contribution

$$
P_{\theta z}^{(r)} \sim P_{\theta z}^{(r 4)}=-\overline{v_{z}^{2}} \frac{\partial V_{\theta}}{\partial z} \sim \omega \frac{r}{\ell} u_{\tau}^{2}=\alpha \frac{U_{j} D}{r \ell} u_{\tau}^{2},
$$

and this behavior is fully satisfied by the computational results, even for strong rotation rates, as shown in Figs. 21(f1)-21(f3).

Now, the contribution of the terms due to rotation in the other components of the production tensor can be readily evaluated,

$$
P_{r r}^{(r)}=2 \overline{v_{r} v_{\theta}} \frac{V_{\theta}}{r} \sim \frac{\alpha^{2}}{R e_{j}^{3 / 4}} \frac{U_{j} D}{r \ell} u_{\tau}^{2} .
$$

For this component, the ratio of the production rate due to rotation to the production rate without rotation is thus of the order $P_{r r}^{(r)} / P_{r r} \sim \alpha^{11 / 5} R e_{j}^{-13 / 10}$. Similarly, for the component $\overline{v_{r} v_{z}}$,

$$
P_{r z}^{(r)}=\overline{v_{\theta} v_{z}} \frac{V_{\theta}}{r} \sim \frac{\alpha^{2}}{\operatorname{Re}_{j}^{3 / 4}} \frac{U_{j} D}{r \ell} u_{\tau}^{2}
$$

such that $P_{r z}^{(r)} / P_{r z}$ is of the order $\alpha^{11 / 5} R e_{j}^{-13 / 10}$ as well. For the azimuthal component $\overline{v_{\theta}^{2}}$,

$$
P_{\theta \theta}^{(r)}=-2 \overline{v_{r} v_{\theta}} \frac{\partial V_{\theta}}{\partial r}-2 \overline{v_{\theta} v_{z}} \frac{\partial V_{\theta}}{\partial z} \sim\left(\frac{\ell}{r}+1\right) \frac{\alpha^{2}}{R e_{j}^{3 / 4}} \frac{U_{j} D}{\ell^{2}} u_{\tau}^{2} \sim \frac{\alpha^{2}}{R e_{j}^{3 / 4}} \frac{U_{j} D}{\ell^{2}} u_{\tau}^{2} .
$$

For this component, the situation is different, since, in the absence of rotation, the production term $P_{\theta \theta}$ is small compared to $P$ (and negative), such that $\overline{v_{\theta}^{2}}$ essentially receives energy from other terms (redistribution, convection, diffusion), and these terms are of the same order of magnitude as production $P$. The correct criterion to evaluate the influence of rotation on $\overline{v_{\theta}^{2}}$ is then the ratio

$$
P_{\theta \theta}^{(r)} / P \sim \alpha^{12 / 5} R e_{j}^{-11 / 10} .
$$

It can be seen in Figs. 21(a1) and 21(b1) that, when rotation is increased, the term $P_{\theta \theta}^{(r 2)}$ rapidly becomes much stronger than $P_{r r}$, such that the turbulent anisotropy is completely modified and, consequently, redistribution mechanisms are reversed, as can be seen in Figs. 22(a1), 22(b1), and 22(c1), energy being redistributed from $\overline{v_{\theta}^{2}}$ to $\overline{v_{r}^{2}}$ and $\overline{v_{z}^{2}}$. In Fig. 22, columns 2 and 3, it is observed that this energy transfer mechanism (production of $\overline{v_{\theta}^{2}}$ and redistribution towards other components) by far becomes dominant, and even overwhelms the production in the outer layer. The order of magnitude analysis leading to Eq. (88) thus shows that the value of $\alpha$ for which $P_{\theta \theta}^{(r 2)}$ becomes significant scales with $R e_{j}^{11 / 24}$; our numerical results suggest that the threshold is about $\alpha \approx 0.01 R e_{j}^{11 / 24}$.

Figure 23 compares the profiles of the azimuthal Reynolds stress component $\overline{v_{\theta}^{2}}$ given by the models to the experimental data. It is seen that when the disk is rotating, $\overline{v_{\theta}^{2}}$ is increased up to a factor of 60, a trend that is reproduced by the EB-RSM, although it is underestimated for the strong 

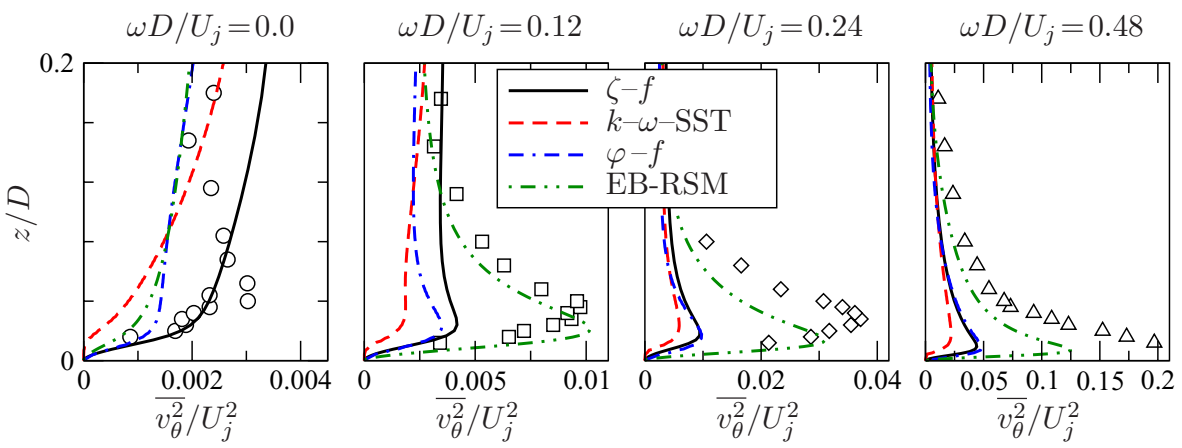

FIG. 23. Profiles of $\overline{v_{\theta}^{2}}$ at $r / D=5.80$ for the 4 rotation rates.

rotation rates. It is worth mentioning that $\overline{v_{\theta}^{2}}$ is underestimated in the case without rotation $\left(\omega D / U_{j}\right.$ $=0$ ), which is related to the underestimation of $V_{r}$ (Figs. 3(g)-3(i)). To say the obvious, this effect of rotation on turbulence cannot be reproduced by linear eddy-viscosity models, since they estimate $\overline{v_{\theta}^{2}}$ by

$$
\overline{v_{\theta}^{2}}=-2 v_{t} \frac{V_{r}}{r}+\frac{2}{3} k,
$$

and are thus completely insensitive to rotation: the increase of $\overline{v_{\theta}^{2}}$ with rotation observed in Fig. 23 is only a consequence of the increase of $V_{r}$ due to the centrifugal acceleration.

One could expect from the above analysis that, when the rotation rate is further increased, $P_{r r}^{(r)}$ and $P_{r z}^{(r)}$ will become dominant in $P_{r r}$ and $P_{r z}$, respectively, but it is worth pointing out that the analysis does not hold anymore for such rotation rates, since the Reynolds stress budgets are strongly modified by the emergence of $P_{\theta \theta}^{(r 2)}$. The profiles of the production terms in Fig. 22 (plots (a1)-(a3) and (e1)-(e3)) indeed indicate that the rotation-induced production terms in $P_{r r}$ and $P_{r z}$ actually never become significant.

\section{Scenario}

The results obtained in Secs. V C 1 and V C 2 suggest the following scenario for the appearance of rotational effects in the wall jet when the rotation rate is gradually increased, or, equivalently, when a fluid particle moves along the $r$-axis above the rotating disk:

(i) As long as

$$
\alpha<0.01 \operatorname{Re}_{j}^{11 / 24},
$$

the dynamics of the flow is dominated by the wall jet: $V_{\theta}, \overline{v_{r} v_{\theta}}$, and $\overline{v_{\theta} v_{z}}$ are non-zero, but they do not have a role in the dynamics of the flow in the radial direction. The azimuthal boundary layer is only superimposed onto the wall jet. In particular, the turbulent energy transfer mechanisms (from the mean flow to the turbulence and among Reynolds stress components by redistribution) are not modified. This regime is called Superposition regime in Fig. 24.

(ii) For higher values of $\alpha, P_{\theta \theta}^{(r 2)}$ becomes the dominant production term in the inner layer, such that redistribution is inverted (now from $\overline{v_{\theta}^{2}}$ to the others). The structure of the inner layer is completely modified, and now resemble that of a boundary layer over a rotating disk in still air. However, the thickness $\ell$ of this azimuthal boundary layer is not sufficient to directly affect the outer layer, which remains typical of a radial wall jet. Therefore, the structure of the flow, and, in particular, the turbulent anisotropy is complex and challenging for turbulence models. This regime is called the Interaction regime in Fig. 24. This regime is observed as long as the centrifugal acceleration remains negligible in the radial momentum equation, and experimental 


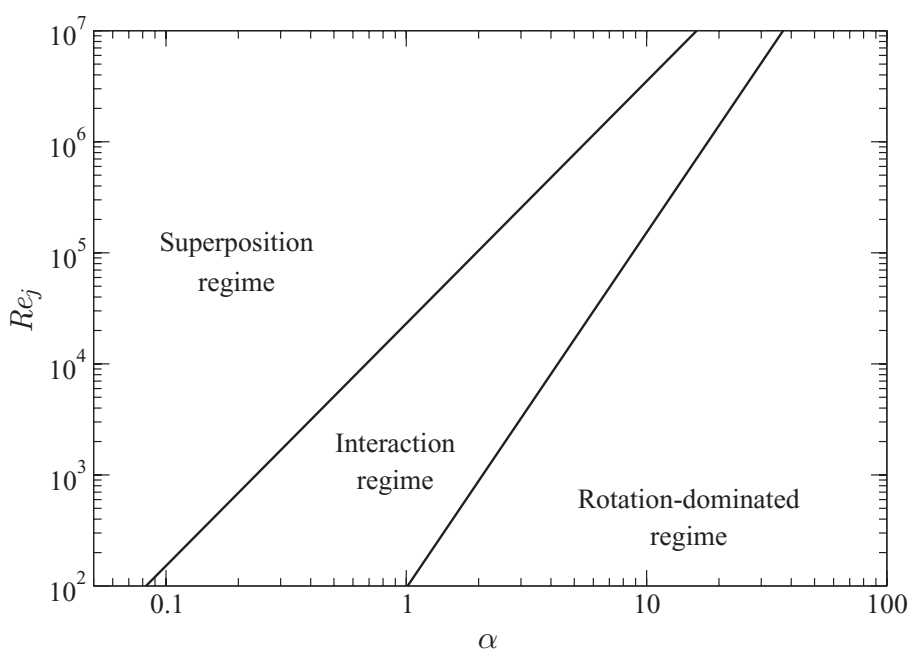

FIG. 24. Different regimes delimited by Eqs. (90) and (91) in the plane $\alpha-R e_{j}$.

and numerical results suggest a regime transition at about

$$
\alpha<0.25 \operatorname{Re}_{j}^{5 / 16} .
$$

Note that this regime does not exist for $R e_{j}>4 \times 10^{9}$.

(iii) For higher rotation rates, centrifugal acceleration dominates the mean momentum budget in the inner layer, but does not affect directly the outer layer that does not rotate. However, the radial acceleration of the inner layer is at the origin of a suction and, in turn, an acceleration of the outer layer, by mass conservation. In this regime, called Rotation-dominated regime in Fig. 24, the mean flow is driven by centrifugal acceleration, independently of turbulence, such that all the turbulence models, including linear eddy-viscosity models, are able to reproduce the effect of rotation.

\section{CONCLUSIONS}

The case of a round jet impinging perpendicularly onto a rotating, heated disc at Reynolds number $R e_{j}=14500$ and the orifice-to-plate distance $H / D=5$ was investigated by making use of a combination of theoretical analysis, turbulence model computations and available experimental data. The focus was on the influence of the rotation rate on the mean flow, turbulence, and heat transfer in the axisymmetric wall jet developing along the disk outside the impingement region, for the four rotation rates documented in the available experiments.

The analysis of the results obtained with the different models shows that an accurate prediction of the velocity and turbulent fields in this configuration is a very challenging task. The overestimation of the spreading of the free jet before it impinges on the wall deteriorates the predictions of the impingement region, in particular the Nusselt number, and of the surrounding wall jet. The second moment closure fails to show any superiority in this respect. This misprediction of the free jet was shown to be due to an excessive turbulent mixing, observed for all the turbulence models applied herein.

In the non-rotational case, it was shown that the inner and outer layers of the wall jet are mutually in equilibrium, and approach a self-similar state with a power law variation of the jet thickness and the flow rate. This behavior is well reproduced by the Reynolds stress and eddy-viscosity models, although the $\varphi-f$ model fails to perfectly reach self-similarity.

The influence of rotation on the wall jet was investigated in detail making use of an order of magnitude analysis. The appearance of a rotation-induced boundary layer, which is well documented in the case of a disk rotating in still air, does not directly perturb the outer layer, even for strong 
rotation rates, since the thickness of this layer always remains small compared to the wall-jet thickness. Therefore, the mechanism responsible for the drastic modification of the flow when the rotation rate is increased is an acceleration and skewing of the inner layer that leads to a suction of the outer layer by mass conservation. A major consequence of this mechanism is that, contrary to the mean flow, the turbulent field in the outer layer is weakly affected by the rotational effects, such that entrainment remains independent of the rotation rate. This behavior explains why, as conjectured by Minagawa and Obi, ${ }^{11}$ the wall-jet thickness varies as the inverse of the non-dimensional parameter $\alpha=\left(r^{2} \omega\right) /\left(U_{j} D\right)$. However, it was shown that in order to observe this scaling in the experimental and numerical results, the wall-jet thickness must be defined as the integral of the non-dimensional radial velocity in the wall-normal direction, and not by the location of the velocity peak: these definitions are not equivalent in the rotating case, since self-similarity is broken.

Eddy-viscosity models are not able to reproduce this behavior, because of the algebraic dependence of the Reynolds stresses on the mean field. On the contrary, scaling properties obtained for strong rotation rates, such as the increase of the peak velocity and the Nusselt number, are reproduced independently of the turbulence model, since they are linked to the predominance of the centrifugal acceleration in the momentum equation.

The order of magnitude analysis supports a scenario for the gradual increase of the influence of rotation with the rotation rate or the distance from the rotation axis, based on the two non-dimensional parameters $\alpha$ and $R e_{j}$. For low rotation rates, such that $\alpha<0.01 R e_{j}^{11 / 24}$, the flow is in a superposition regime, in which the rotation-induced boundary layer does not significantly affect the self-similar wall jet. For intermediate rotation rates, $0.01 R e_{j}^{11 / 24}<\alpha<0.25 R e_{j}^{5 / 16}$, an Interaction regime is observed, in which the inner layer resemble an Ekman layer, while the outer layer remains weakly perturbed. Finally, for strong rotation rates, $\alpha>0.25 R e_{j}^{5 / 16}$, in the rotation-dominated regime, centrifugal acceleration becomes dominant and causes an intensification and thinning of the jet.

\section{APPENDIX: ORDERS OF MAGNITUDE OF $\overline{v_{r} v_{\theta}}$ AND $\overline{v_{\theta} v_{z}}$ IN THE INNER LAYER}

Even though turbulence is not in equilibrium, it can be assumed that the production and dissipation rates are of the same order of magnitude, $P \sim \varepsilon$, which is confirmed by Fig. 22. Therefore, the turbulent time scale, $\tau=u^{2} / \varepsilon$ is of the order of $u^{2} / P$, which can be obtained from Eqs. (78) and (79), such that

$$
\tau \sim \frac{1}{R e_{j}^{3 / 4}} \frac{r^{2}}{U_{j} D}
$$

Considering that turbulence is driven by a single time scale $\overline{v_{r} v_{\theta}} / P_{r \theta}^{(r)} \sim u^{2} / P \sim \tau$ yields

$$
P_{r \theta}=P_{r \theta}^{(r)} \sim \frac{\overline{v_{r} v_{\theta}}}{\tau} \sim \operatorname{Re}_{j}^{3 / 4} \overline{v_{r} v_{\theta}} \frac{U_{j} D}{r^{2}} .
$$

Introducing this relation in Eq. (83) leads to

$$
\operatorname{Re}_{j}^{3 / 4} \overline{v_{r} v_{\theta}} \frac{U_{j} D}{r^{2}} \sim \frac{1}{\operatorname{Re}_{j}^{1 / 8}} \overline{v_{r} v_{\theta}} \frac{U_{j} D}{r L}+u_{\tau}^{2} \omega \frac{r}{\ell}+R e_{j}^{3 / 4} \overline{v_{\theta} v_{z}} \frac{U_{j} D}{r^{2}} .
$$

The first term in the right-hand side is smaller than the left-hand side by a factor of $R e_{j}^{7 / 8} L / r$, such that

$$
\overline{v_{r} v_{\theta}} \sim \frac{1}{R e_{j}} U_{j} D \frac{r}{\ell} \omega+\overline{v_{\theta} v_{z}} .
$$

The same analysis applied to $P_{\theta z}^{(r)}$

$$
P_{\theta z}^{(r)}=\underbrace{P_{\theta z}^{(r 1)}}_{\sim \overline{v_{r} v_{\theta}} \frac{1}{R e_{j}}}+\underbrace{P_{\theta z}^{(r 2)}}_{\sim \overline{v_{\theta} v_{z}} \frac{1}{L^{2} e_{j}^{1 / 8}} \frac{U_{j} D}{r^{2}}}+\underbrace{P_{\theta z}^{(r 3)}}_{\sim u_{\tau}^{2} \omega}+\underbrace{P_{\theta z}^{(r 4)}}_{\sim u_{\tau}^{2} \omega_{\frac{r}{\ell}}},
$$


yields

$$
\overline{v_{\theta} v_{z}}=\frac{1}{R e_{j}^{7 / 4}} \frac{r^{2}}{L^{2}} \overline{v_{r} v_{\theta}}+\frac{1}{R e_{j}} U_{j} D \frac{r}{\ell} \omega,
$$

such that the system of two equations (A4)+(A6) can be easily solved, leading to

$$
\overline{v_{r} v_{\theta}} \sim \overline{v_{\theta} v_{z}} \sim \frac{1}{R e} U_{j} D \frac{r \omega}{\ell}=\frac{\alpha}{R e_{j}^{3 / 4}} \frac{r}{\ell} u_{\tau}^{2} .
$$

Finally, introducing this relation into Eq. (83) shows that $P_{r \theta}^{(r 3)}$ and $P_{r \theta}^{(r 5)}$ are of the same order of magnitude and are dominant in $P_{r \theta}^{(r)}$,

$$
P_{r \theta}^{(r)} \sim P_{r \theta}^{(r 3)}+P_{r \theta}^{(r 5)}=-\underbrace{\overline{v_{r} v_{z}} \frac{\partial V_{\theta}}{\partial z}}_{\sim \omega_{\bar{l}}^{\frac{r}{l}} u_{\tau}^{2}}-\underbrace{\overline{v_{\theta} v_{z}} \frac{\partial V_{r}}{\partial z}}_{\sim \omega_{\bar{l}}^{\frac{r}{l}} u_{\tau}^{2}} \sim \alpha \frac{U_{j} D}{r \ell} u_{\tau}^{2} .
$$

${ }^{1}$ M. Hadžiabdić and K. Hanjalić, "Vortical structures and heat transfer in a round impinging jet," J. Fluid Mech. 596, 221-260 (2008).

${ }^{2}$ Y.-M. Chen, W.-T. Lee, and S.-J. Wu, "Heat (mass) transfer between an impinging jet and a rotating disk," Heat Mass Transfer 34, 195-201 (1998).

${ }^{3}$ A. Benim, K. Ozkan, M. Cagan, and D. Gunes, "Computational investigation of turbulent jet impinging onto rotating disk," Int. J. Numer. Methods Heat Fluid Flow 17, 284-301 (2005).

${ }^{4}$ S. Harmand, J. Pellé, S. Poncet, and I. Shevchuk, "Review of fluid flow and convective heat transfer within rotating disk cavities with impinging jet," Int. J. Therm. Sci. 67, 1-30 (2013).

${ }^{5}$ S. Brodersen, D. Metzger, and H. Fernando, "Flows generated by the impingement of a jet on a rotating surface: Part I Basic flow patterns," Trans. ASME J. Fluid Eng. 118, 61-67 (1996).

${ }^{6}$ C. Popiel and L. Boguslawski, "Local heat transfer from a rotating disk in an impinging round jet," J. Heat Transfer 108, 357-364 (1986).

${ }^{7}$ T. von Kármán, “Über laminare und turbulente Reibung,” Z. Angew. Math. Mech. 1, 233-252 (1921).

${ }^{8}$ E. Truckenbrodt, "Die turbulente Strömung an einer angeblasenen rotierenden Scheibe," Z. Angew. Math. Mech. 34, 150-162 (1954).

${ }^{9}$ L. A. Dorfman, Hydrodynamic Resistance and the Heat Loss of Rotating Solids (Oliver \& Boyd, Edinburgh, 1963).

${ }^{10}$ M. Itoh and M. Okada, "An experimental study of the radial wall jet on a rotating disk," Exp. Therm. Fluid Sci. 17, 49-56 (1998).

${ }^{11}$ Y. Minagawa and S. Obi, "Development of turbulent impinging jet on a rotating disk," Int. J. Heat Fluid Flow 25, 759-766 (2004).

${ }^{12}$ T. Astarita and G. Cardone, "Convective heat transfer on a rotating disk with a centred impinging round jet," Int. J. Heat Mass Transfer 51, 1562-1572 (2008).

${ }^{13}$ H. Steiner, S. Jakirlić, G. Kadavelil, R. Manceau, S. Sarić, and G. Brenn, "13th ERCOFTAC Workshop on refined turbulence modelling, 25-26th September, 2008, Graz University of Technology, Austria," ERCOFTAC Bulletin (ERCOFTAC, Brussels, 2009), Vol. 79, pp. 22-27.

${ }^{14}$ B. Ničeno, "An unstructured parallel algorithm for large eddy and conjugate heat transfer simulations," Ph.D. thesis, Delft University of Technology, 2001.

${ }^{15}$ B. Ničeno and K. Hanjalić, "Unstructured large-eddy- and conjugate heat transfer simulations of wall-bounded flows," Modeling and Simulation of Turbulent Heat Transfer (WIT Press, 2004).

${ }^{16}$ F. Archambeau, N. Méchitoua, and M. Sakiz, "Code Saturne: A finite volume code for the computation of turbulent incompressible flows: Industrial applications," Int. J. Finite Vol. 1 (2004), Electronical edition: http://averoes.math.univ-paris13.fr/html.

${ }^{17} \mathrm{~K}$. Hanjalić, M. Popovac, and M. Hadžiabdić, "A robust near-wall elliptic relaxation eddy-viscosity turbulence model for CFD,” Int. J. Heat Fluid Flow 25, 1047-1051 (2004).

${ }^{18}$ F. R. Menter, "Two-equation eddy-viscosity turbulence models for engineering applications," AIAA J. 32, 1598-1605 (1994).

${ }^{19}$ D. R. Laurence, J. C. Uribe, and S. V. Utyuzhnikov, “A robust formulation of the $v^{2}-$ f model," Flow Turbul. Combust. 73, 169-185 (2005).

${ }^{20}$ R. Manceau and K. Hanjalić, "Elliptic blending model: A new near-wall Reynolds-stress turbulence closure," Phys. Fluids 14, 744-754 (2002).

${ }^{21}$ F. Dehoux, Y. Lecocq, S. Benhamadouche, R. Manceau, and L.-E. Brizzi, "Algebraic modeling of the turbulent heat fluxes using the elliptic blending approach: Application to forced and mixed convection regimes," Flow Turbul. Combust. 88, 77-100 (2012).

${ }^{22}$ C. G. Speziale, S. Sarkar, and T. B. Gatski, "Modeling the pressure-strain correlation of turbulence: An invariant dynamical system approach," J. Fluid Mech. 227, 245-272 (1991).

${ }^{23}$ J. Baughn and S. Shimizu, "Heat transfer measurements from a surface with uniform heat flux and an impinging jet," J. Heat Transfer 111, 1096-1098 (1989). 
${ }^{24}$ P. A. Durbin, "On the $k-3$ stagnation point anomaly," Int. J. Heat Fluid Flow 17, 89-90 (1996).

${ }^{25}$ D. Cooper, D. C. Jackson, B. E. Launder, and G. X. Liao, "Impinging jet studies for turbulence model assessment-I. Flow-field experiments," Int. J. Heat Mass Transfer 36, 2675-2684 (1993).

${ }^{26}$ S. B. Pope, "An explanation of the turbulent round-jet/plane-jet anomaly," AIAA J. 16, 279-281 (1978).

${ }^{27}$ M. Glauert, "The wall jet," J. Fluid Mech. 1, 625-643 (1956).

${ }^{28}$ B. Launder and W. Rodi, "The turbulent wall jet: Measurements and modeling," Annu. Rev. Fluid Mech. 15, 429-459 (1983).

${ }^{29}$ P. Bakke, “An experimental investigation of a wall jet,” J. Fluid Mech. 2, 467-472 (1957).

${ }^{30}$ P. Bradshaw and E. Love, "The normal impingement of a circular air jet on a flat surface," Reports and Memoranda 3205 (Ministry of Aviation, Aeronautical Research Council, London, UK, 1959).

${ }^{31}$ A. Verhoff, "The two-dimensional turbulent wall jet with and without external free stream," Report No. 626 (Aeronautical Engineering Department, Princeton University, Princeton, NJ, USA, 1963).

${ }^{32}$ W. George, H. Abrahamsson, J. Eriksson, R. Karlsson, L. Lofdahl, and M. Wosnik, "A similarity theory for the turbulent plane wall jet without external stream,” J. Fluid Mech. 425, 367-411 (2000).

${ }^{33}$ G. Barenblatt, A. Chorin, and V. Prostokishin, "The turbulent wall jet: A triple-layered structure and incomplete similarity," Proc. Natl. Acad. Sci. U.S.A. 102, 8850-8853 (2005).

${ }^{34}$ H. Tennekes and J. L. Lumley, A First Course in Turbulence (MIT Press, 1972).

${ }^{35}$ C. Jayatilleke, "The influence of Prandtl number and surface roughness on the resistance of the laminar sub-layer to momentum and heat transfer," Prog. Heat Mass Transfer 1, 193-329 (1969).

${ }^{36}$ B. Kader and A. Yaglom, "Heat and mass transfer laws for fully turbulent wall flows," Int. J. Heat Mass Transfer 15, 2329-2351 (1972).

${ }^{37}$ V. Arpaci and P. Larsen, "Heat transfer prediction," Convection Heat Transfer (Prentice-Hall, Englewood Cliffs, NJ, 1984), Chap. 11, pp. 434-462.

${ }^{38}$ H. Schlichting, Boundary Layer Theory, 7th ed. (McGraw-Hill, Inc., 1979).

${ }^{39}$ G. Cardone, T. Astarita, and G. Carlomagno, "Heat transfer measurements on a rotating disk," Int. J. Rotating Mach. 3, 1-9 (1997). 